This is an electronic reprint of the original article. This reprint may differ from the original in pagination and typographic detail.

Author(s): Satuła, W.; Bączyk, P.; Dobaczewski, Jacek; Konieczka, M.

Title: No-core configuration-interaction model for the isospin- and angular-momentumprojected states

Year: $\quad 2016$

Version:

Please cite the original version:

Satuła, W., Bączyk, P., Dobaczewski, J., \& Konieczka, M. (2016). No-core

configuration-interaction model for the isospin- and angular-momentum-projected states. Physical Review C, 94(2), Article 024306.

https://doi.org/10.1103/PhysRevC.94.024306

All material supplied via JYX is protected by copyright and other intellectual property rights, and duplication or sale of all or part of any of the repository collections is not permitted, except that material may be duplicated by you for your research use or educational purposes in electronic or print form. You must obtain permission for any other use. Electronic or print copies may not be offered, whether for sale or otherwise to anyone who is not an authorised user. 


\title{
No-core configuration-interaction model for the isospin- and angular-momentum-projected states
}

\author{
W. Satuła, ${ }^{1,2}$ P. Bączyk, ${ }^{1}$ J. Dobaczewski, ${ }^{1,2,3,4}$ and M. Konieczka ${ }^{1}$ \\ ${ }^{1}$ Institute of Theoretical Physics, Faculty of Physics, University of Warsaw, ul. Pasteura 5, PL-02-093 Warsaw, Poland \\ ${ }^{2}$ Helsinki Institute of Physics, P.O. Box 64, FI-00014 University of Helsinki, Finland \\ ${ }^{3}$ Department of Physics, University of York, Heslington, York YO10 5DD, United Kingdom \\ ${ }^{4}$ Department of Physics, P.O. Box 35 (YFL), University of Jyväskylä, FI-40014 Jyväskylä, Finland
}

(Received 14 January 2016; published 4 August 2016)

\begin{abstract}
Background: Single-reference density functional theory is very successful in reproducing bulk nuclear properties like binding energies, radii, or quadrupole moments throughout the entire periodic table. Its extension to the multireference level allows for restoring symmetries and, in turn, for calculating transition rates.

Purpose: We propose a new variant of the no-core-configuration-interaction (NCCI) model treating properly isospin and rotational symmetries. The model is applicable to any nucleus irrespective of its mass and neutronand proton-number parity. It properly includes polarization effects caused by an interplay between the long- and short-range forces acting in the atomic nucleus.

Methods: The method is based on solving the Hill-Wheeler-Griffin equation within a model space built of linearly dependent states having good angular momentum and properly treated isobaric spin. The states are generated by means of the isospin and angular-momentum projection applied to a set of low-lying (multi)particle-(multi)hole deformed Slater determinants calculated using the self-consistent Skyrme-Hartree-Fock approach.

Results: The theory is applied to calculate energy spectra in $N \approx Z$ nuclei that are relevant from the point of view of a study of superallowed Fermi $\beta$ decays. In particular, a new set of the isospin-symmetry-breaking corrections to these decays is given.

Conclusions: It is demonstrated that the NCCI model is capable of capturing main features of low-lying energy spectra in light and medium-mass nuclei using relatively small model space and without any local readjustment of its low-energy coupling constants. Its flexibility and a range of applicability makes it an interesting alternative to the conventional nuclear shell model.
\end{abstract}

DOI: 10.1103/PhysRevC.94.024306

\section{INTRODUCTION}

Atomic nucleus is a self-bound finite system composed of neutrons and protons that interact by means of short-range, predominantly isospin-symmetry-conserving strong force and long-range isospin-symmetry-breaking Coulomb force. In studies of phenomena related to the isospin-symmetry violation in nuclei, capturing a delicate balance between these two forces is of utmost importance. This is particularly true when evaluating the isospin-symmetry-breaking (ISB) corrections to superallowed $\beta$ decays between isobaric analog states, $\left[I=0^{+}, T=1\right] \longrightarrow\left[I=0^{+}, T=1\right]$.

Such $\beta$ decays currently offer the most precise data that give estimates of the vector coupling constant $G_{V}$ and leading element $V_{u d}$ of the Cabibbo-Kobayashi-Maskawa (CKM) flavor-mixing matrix [1,2]. The uncertainty of $V_{u d}$ extracted from the superallowed $\beta$ decays is almost an order of magnitude smaller than that from neutron or pion decays [3]. To test the weak-interaction flavor-mixing sector of the Standard Model of elementary particles, such precision is critical, because it allows us to verify the unitarity of the CKM matrix, violation of which may signal new physics beyond the Standard Model; see Ref. [4] and references cited therein.

The isospin impurity of the nuclear wave function-a measure of the ISB-is small. It varies from a fraction of a percent, in ground states of even-even $N=Z$ light nuclei, to about six percent in the heaviest known $N=Z$ system,

${ }^{100} \mathrm{Sn}$ [5]. Nevertheless, its microscopic calculation poses a real challenge to theory. The reason is that the isospin impurity originates from the long-range Coulomb force that polarizes the entire nucleus and can be, therefore, calculated only within so-called no-core approaches. In medium and heavy nuclei, it narrows the possible microscopic models to those rooted within the nuclear density functional theory (DFT) $[6,7]$.

The absence of external binding requires that the nuclear DFT be formulated in terms of intrinsic, and not laboratory densities. This, in turn, leads to the spontaneous breaking of fundamental symmetries of the nuclear Hamiltonian, including the rotational and isospin symmetries, which in finite systems must be restored. Fully quantal calculations of observables, such as matrix elements of electromagnetic transitions or $\beta$-decay rates, require symmetry restoration. In most of the practical applications, this is performed with the aid of the generalized Wick's theorem [8]. Its use, however, leads to the energy density functionals (EDFs) being expressed in terms of the so-called transition densities, that is, to a multireference (MR) DFT. Unfortunately, the resulting MR EDFs are, in general, singular and require regularization, which still lacks satisfactory and practical solution; see, e.g., Refs. [9-11]. An alternative way of building a nonsingular MR theory, the one that we use in the present work, relies on employing the EDFs derived from a true interaction, which then acquires a role of the EDF generator [12]. The results presented here were obtained using in this role the density-independent Skyrme interaction SV [13], augmented by the tensor terms $\left(\mathrm{SV}_{T}\right)$ [11]

Over the last few years we have developed the MR DFT approach based on the angular-momentum and/or isospin 
projections of single Slater determinants. The model, below referred to as static, was specifically designed to treat rigorously the conserved rotational symmetry and, at the same time, tackle the explicit Coulomb-force mixing of good-isospin states. This unique approach allowed us to determine the isospin impurities in $N \approx Z$ nuclei [5] and ISB corrections to superallowed $\beta$-decay matrix elements $[14,15]$.

In this paper, following upon preliminary results announced at several conferences [16-18], we introduce a next-generation dynamic variant of the approach, which we call the nocore configuration-interaction (NCCI) model. It constitutes a natural extension of the static MR DFT model, and allows for mixing states that are projected from different self-consistent Slater determinants representing low-lying (multi)particle(multi)hole excitations. Technically, the model is analogous to the generator-coordinate-method (GCM) mixing of symmetryprojected states; see, e.g., Ref. [19]. However, the GCM pertains to mixing continuous sets of states, and thus builds collective states of the system, whereas NCCI involves mixing of discrete configurations.

In quantum chemistry such a method is commonly known under the name of configuration interaction $(\mathrm{CI})$, where the interaction means mixing of different electronic configurations. In nuclear physics, models of this type go by the name of the shell model (SM), whereupon all configurations within a specific valence shell are considered. In recent years, in relatively light nuclei, a no-core variant of the shell model (NCSM) was very successfully implemented [20]. Our approach combines the no-core aspect of the NCSM and the mixing aspect of the CI, and, by using sets of selected DFT configurations, it is not limited to light nuclei.

In nuclear physics the name CI is seldom used, and, in our opinion, it is meaningful to import it from quantum chemistry, as exemplified in the name proposed for our model. In spite of apparent similarities, there are also differences between the SM and CI methods. Indeed, the SM indiscriminately uses expansions on large bases (most often of the harmonicoscillator states), which are unrelated with the interaction used or properties of the system. As a result, the SM is bound to use millions or billions of states, which strongly limits its applicability range. On the other hand, the CI uses bases of approximate states of the very system that is being described. In this way, the most important correlations are already built in into the basis states, and thus much smaller bases can be used. At present, another difference between the NCSM and NCCI methods is in the fact that the former is often rooted in true nucleon interactions, and thus belongs to the class of $a b$ initio methods, whereas the latter still uses phenomenological density functionals. This difference may eventually disappear once $a b$ initio nuclear DFT methods are developed in analogy to those available for electronic systems [21].

There are several cases when, to perform reliable calculations, the NCCI approach is indispensable. One of the most important ones relates to different possible shape-current orientations, which within the static variant of the model appear in odd-odd nuclei [15]. The configuration mixing is also needed to resolve the issue of unphysical ISB corrections to the analogous states of the $A=38$ isospin triplet $[14,15]$.
The states that are mixed have good angular momenta and, at the same time, include properly evaluated Coulomb isospin mixing; hence, the extended model treats hadronic and Coulomb interactions on the same footing. The model is based on a truncation scheme dictated by the self-consistent deformed Hartree-Fock (HF) solutions, and can be used to calculate spectra, transitions, and $\beta$-decay rates in any nucleus, irrespective of its even or odd neutron and proton numbers.

We begin by giving in Sec. II a short overview of the theoretical framework of our NCCI model. In Sec. III, a new set of the ISB corrections to the canonical set of superallowed $\beta$ decay is presented. As compared to our previous results [15], the new set includes mixing of reference states corresponding to different shape-current orientations in oddodd $N=Z$ nuclei. In Sec. IV, applications involving mixing of several low-energy (multi)particle-(multi)hole excitations are discussed. Here, we determined low-spin energy spectra in selected nuclei relevant to high-precision tests of the weakinteraction flavor-mixing sector of the Standard Model. The calculations were performed for ${ }^{6} \mathrm{Li}$ and ${ }^{8} \mathrm{Li}$ nuclei (Sec. IV A), $A=38 \mathrm{Ar}, \mathrm{K}$, and Ca nuclei (Sec. IV B), ${ }^{42} \mathrm{Sc}$ and ${ }^{42} \mathrm{Ca}$ nuclei (Sec. IV C), and ${ }^{62} \mathrm{Ga}$ and ${ }^{62} \mathrm{Zn}$ nuclei (Sec. IV D; Summary and perspectives are given in Sec. V).

\section{THE NO-CORE CONFIGURATION-INTERACTION MODEL}

The static model developed in our previous works [15,22] involved the isospin and angular momentum projections (after variation) of a single Slater determinant, followed by a rediagonalization of the Coulomb force, so as to account properly for the isospin mixing. Here we extend the model towards a variant, in which we allow for a mixing of states projected from different low-lying (multi)particle-(multi)hole Slater determinants $\varphi_{i}$ with the mixing matrix elements derived from the same Hamiltonian that is used to calculate them.

The computational scheme of our NCCI model is sketched in Fig. 1. It proceeds in four major steps:

(i) First, a set of relevant low-lying (multi)particle(multi)hole HF states $\left\{\varphi_{i}\right\}$ is calculated along with their HF energies $e_{i}^{(\mathrm{HF})}$. States $\left\{\varphi_{i}\right\}$ form a subspace of reference states for subsequent projections.

(ii) Second, the projection techniques are applied to the set of reference states $\left\{\varphi_{i}\right\}$, so as to determine the family of states $\left\{\Psi_{T I K}^{(i)}\right\}$ having good isospin $T$, angular momentum $I$, and angular-momentum projection on the intrinsic axis $K$.

(iii) Third, states $\left\{\Psi_{T I K}^{(i)}\right\}$ are mixed, so as to properly take into account the $K$ mixing and Coulomb isospin mixing - this gives the set of good angular-momentum states $\left\{\Psi_{\tilde{T} I \alpha}^{(i)}\right\}$ of the static model $[14,15]$. Here we label them with the dominating values of the isospin $\tilde{T}$, and auxiliary quantum numbers $\alpha$. Note that in this step, the mixing is performed for each configuration $i$ separately (the static model).

(iv) Finally, the results of the dynamic model correspond to mixing nonorthogonal states $\left\{\Psi_{\tilde{T} I \alpha}^{(i)}\right\}$ for all configurations $i$, and for all values of $\tilde{T}$ and $\alpha$. This 

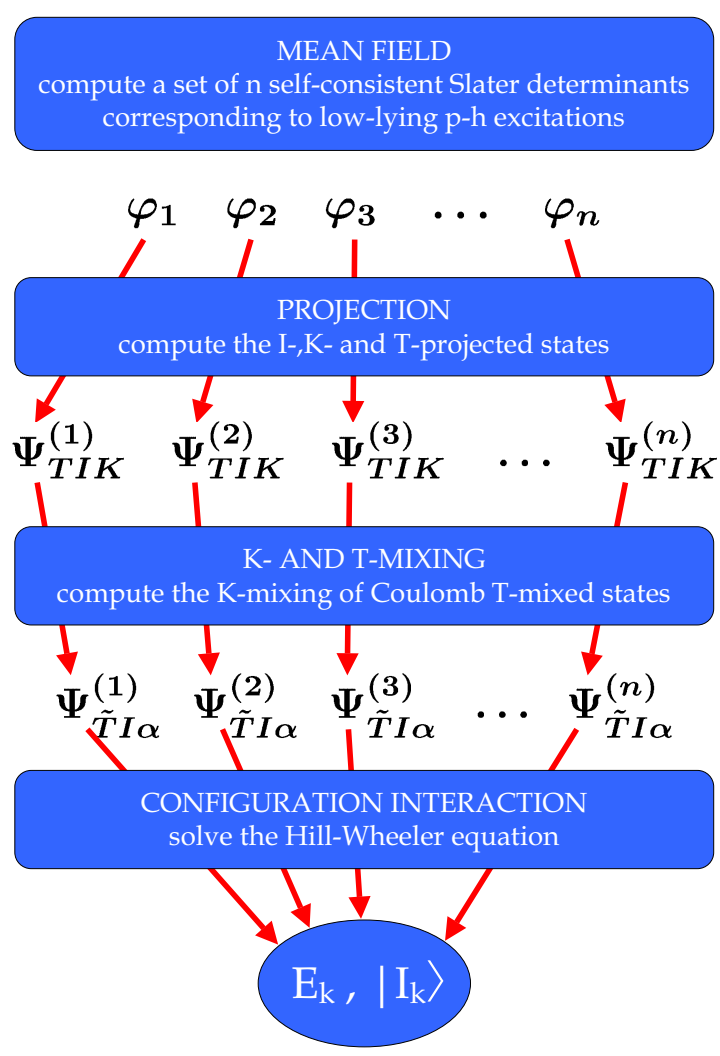

FIG. 1. Computational scheme of the NCCI model. See text for details.

is performed by solving the Hill-Wheeler equation $\mathcal{H}\left|I_{k}\right\rangle=E_{k} \mathcal{N}\left|I_{k}\right\rangle$ [23] in the collective space spanned by the natural states corresponding to sufficiently large eigenvalues of the norm matrix $\mathcal{N}$. This is the same technique that is used in the code to handle the $K$ mixing alone. The method is described in details in Ref. [24].

We note here that all wave functions considered above correspond to good neutron $(N)$, and proton $(Z)$ numbers, and thus to a good third component of the isospin, $T_{z}=\frac{1}{2}(N-Z)$. We also note that the configuration interaction, which is taken into account in the last step, could have also equivalently been performed by directly mixing the projected states $\left\{\Psi_{T I K}^{(i)}\right\}$. The procedure outlined above simply aims to obtain separately the results of the static and dynamic model. The NCCI calculations discussed below were performed using a new version of the HFODD solver [25], which was equipped with the NCCI module. This new implementation was based on the previous versions of the code [24,26,27].

Numerical stability of the method depends on necessary truncations of the model space. In this work, numerically unstable solutions are removed by truncating the natural states corresponding to small eigenvalues of the norm matrix $N$. It means that only the natural states corresponding to the eigenvalues of the norm matrix that are larger than certain externally provided cutoff parameter $\zeta$ are used to build the so-called collective space. Although such a truncation procedure gives reliable values of the energy, a full stability of the method still requires further studies. Other methods, e.g., based on truncating high-energy states $\left\{\Psi_{\tilde{T} I \alpha}^{(i)}\right\}$, or combined methods involving both truncations simultaneously, need to be studied as well.

\section{A NEW SET OF THE ISB CORRECTIONS TO SUPERALLOWED $\beta$ DECAYS}

In this section we present results obtained within the NCCI model, which pertain to removing the uncertainty related to ambiguities in the shape-current orientation. Similar to our previous applications within the static model, the ground states (g.s.) of even-even nuclei, $\left|I=0, T \approx 1, T_{z}= \pm 1\right\rangle$, are approximated by the Coulomb $T$-mixed states,

$$
\begin{aligned}
\left|I=0, T \approx 1, T_{z}= \pm 1\right\rangle & =\Psi_{\tilde{T}=1, I=0, K=0}^{(1)} \\
& =\sum_{T \geqslant 1} c_{T}^{(1)} \Psi_{T, I=0, K=0}^{(1)},
\end{aligned}
$$

which were angular-momentum projected from the MF g.s. $\varphi_{1}$ of the even-even nuclei, obtained in the self-consistent HartreeFock (HF) calculations. States $\varphi_{1}$ are always unambiguously defined by filling in the pairwise doubly degenerate levels of protons and neutrons up to the Fermi level. In the calculations, the Coulomb $T$ mixing was included up to $T=4$.

Within our dynamic model, the corresponding isobaric analogs in $N=Z$ odd-odd nuclei, $\left|I=0, T \approx 1, T_{z}=0\right\rangle$, were approximated by

$$
\left|I=0, T \approx 1, T_{z}=0\right\rangle=\sum_{k=X, Y, Z} \sum_{\tilde{T}=0,1,2} c_{\tilde{T}}^{(k)} \Psi_{\tilde{T}, I=0, K=0}^{(k)} .
$$

The underlying MF states $\varphi_{k}$ were taken as the self-consistent Slater determinants $|\bar{v} \otimes \pi ; k\rangle$ (or $|\nu \otimes \bar{\pi} ; k\rangle$ ) representing the antialigned configurations corresponding to different shape-current orientations $k=X, Y, Z$. Let us recall that the antialigned states are constructed by placing the odd neutron and odd proton in the lowest available time-reversed (or signature-reversed) s.p. orbits. These states are manifestly breaking the isospin symmetry. Using them is the only way to reach the $\left|I=0, T \approx 1, T_{z}=0\right\rangle$ states in odd-odd $N=Z$ nuclei. The reason is that, within a conventional MF approach with separate proton and neutron Slater determinants, these states are not representable by single Slater determinants; see discussion in Ref. [22].

For odd-odd nuclei, mixing coefficients $c_{\tilde{T}}^{(k)}$ in Eq. (2) were determined by solving the Hill-Wheeler equation. In the mixing calculations, we only included states $\Psi_{\tilde{T}, I=0, K=0}^{(k)}$ with dominating isospins of $\tilde{T}=0,1$ and 2 , that is, the Hill-Wheeler equation was solved in the space of six or nine states for axial and triaxial states, respectively. We recall that each of states $\Psi_{\tilde{T}, I=0, K=0}^{(k)}$ contains all Coulomb-mixed good- $T$ components $\Psi_{T, I=0, K=0}^{(k)}$.

The three states corresponding to a given dominating isospin are linearly dependent. One may therefore argue that the physical subspace of the $I=0$ states should be three dimensional. In the calculations, all six or nine eigenvalues 


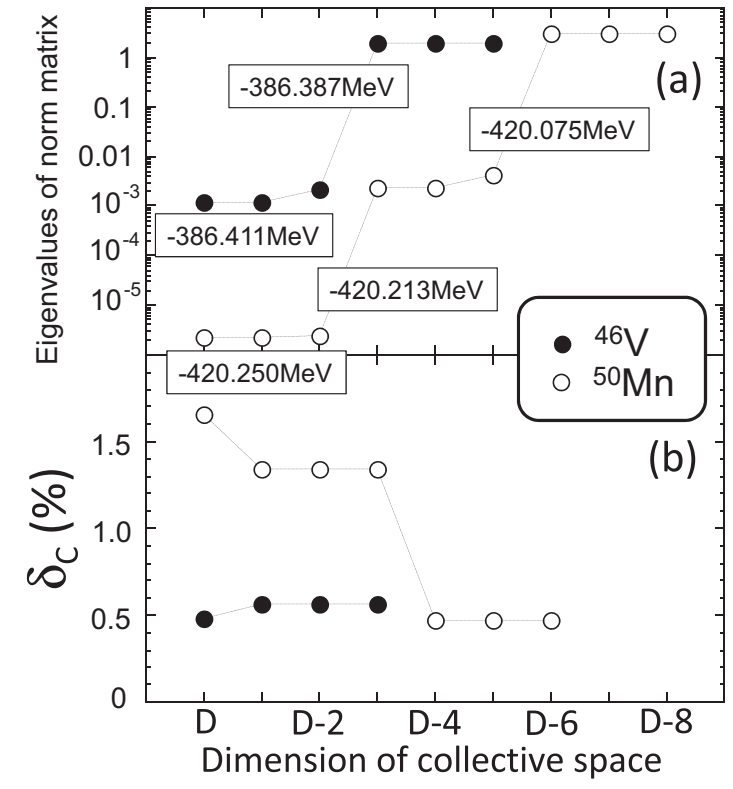

FIG. 2. (a) Eigenvalues of the norm matrix obtained in the NCCI calculations for the $I=0$ states of odd-odd nuclei. Depicted are typical results obtained for two representative examples of axial $\left({ }^{46} \mathrm{~V}\right)$ and triaxial $\left({ }^{50} \mathrm{Mn}\right)$ nuclei. The boxes give values of eigenenergies obtained by including three, six or nine eigenvalues of the norm matrix. (b) Dependence of the ISB corrections to superallowed ${ }^{46} \mathrm{~V} \rightarrow{ }^{46} \mathrm{Ti}$ and ${ }^{50} \mathrm{Mn} \rightarrow{ }^{50} \mathrm{Cr}$ decays on a number of collective states retained in the mixing calculations. Dimension of the collective space decreases from the left- to the right-hand side from $D=9(6)$ in ${ }^{50} \mathrm{Mn}\left({ }^{46} \mathrm{~V}\right)$, respectively.

of the norm matrix $\mathcal{N}$ are nonzero, but the linear dependence of the reference states is clearly reflected in the pattern they form. For two representative examples of axial $\left({ }^{46} \mathrm{~V}\right)$ and triaxial $\left({ }^{50} \mathrm{Mn}\right)$ nuclei, this is depicted in Fig. 2. Note, that the eigenvalues group into two or three sets, each consisting of three similar eigenvalues. Note also that the differences between the sets are large, reaching three-four orders of magnitude. Lower part of the figure illustrates dependence of the calculated ISB corrections $\delta_{\mathrm{C}}$, on a number of the collective states retained in the mixing. As shown, the calculated corrections are becoming stable within a subspace consisting five (or less) highest-norm states. Following this result, we have decided to retain in the mixing calculations only three collective states built upon the three eigenvectors of the norm matrix corresponding to the largest eigenvalues.

Based on this methodology, we calculated the set of the superallowed transitions, which are collected in Tables I and II. Table I shows the empirical $f t$ values, calculated ISB corrections, and so-called nucleus-independent reduced lifetimes,

$$
\mathcal{F} t \equiv f t\left(1+\delta_{\mathrm{R}}^{\prime}\right)\left(1+\delta_{\mathrm{NS}}-\delta_{\mathrm{C}}\right)=\frac{K}{2 G_{\mathrm{V}}^{2}\left(1+\Delta_{\mathrm{R}}^{\mathrm{V}}\right)},
$$

where $\delta_{\mathrm{R}}^{\prime}$ and $\delta_{\mathrm{NS}}$ are the radiative corrections [28]. Errors of $\mathcal{F} t$ include errors of the empirical $f t$ values [29,30], radiative corrections $\delta_{\mathrm{R}}^{\prime}$ and $\delta_{\mathrm{NS}}$ [28], and our uncertainties estimated for the calculated values of $\delta_{\mathrm{C}}$.
TABLE I. Results of calculations performed for nuclei, for which the superallowed transitions have been measured. Listed are empirical $f t$ values [30]; calculated ISB corrections $\delta_{\mathrm{C}}^{(\mathrm{SV})}$ and the corresponding $\mathcal{F} t$ values; empirical corrections $\delta_{\mathrm{C}}^{(\exp )}$ calculated using Eq. (5); contributions coming from the individual transitions to the $\chi^{2}$ budget in the confidence-level test. As in Ref. [30], we give two digits of the calculated errors of the $\mathcal{F} t$ values.

\begin{tabular}{|c|c|c|c|c|c|}
\hline $\begin{array}{l}\text { Parent } \\
\text { nucleus }\end{array}$ & $\begin{array}{l}f t \\
(\mathrm{~s})\end{array}$ & $\begin{array}{l}\delta_{\mathrm{C}}^{(\mathrm{SV})} \\
(\%)\end{array}$ & $\begin{array}{l}\mathcal{F} t \\
(\mathrm{~s})\end{array}$ & $\begin{array}{l}\delta_{\mathrm{C}}^{(\exp )} \\
(\%)\end{array}$ & $\chi_{i}^{2}$ \\
\hline$T_{z}=-1:$ & $3042(4)$ & $0.579(87)$ & $3064.5(52)$ & $0.37(15)$ & 3.5 \\
\hline${ }^{14} \mathrm{O}$ & $3042.3(27)$ & $0.303(30)$ & $3072.3(33)$ & $0.36(6)$ & 0.0 \\
\hline${ }^{22} \mathrm{Mg}$ & $3052(7)$ & $0.270(41)$ & $3081.4(72)$ & $0.62(23)$ & 1.4 \\
\hline${ }^{34} \mathrm{Ar}$ & 3053(8) & $0.87(13)$ & $3063.6(91)$ & $0.63(27)$ & 1.3 \\
\hline \multicolumn{6}{|l|}{$T_{z}=0$} \\
\hline${ }^{34} \mathrm{Cl}$ & $3049.4(12)$ & $0.75(11)$ & $3067.6(38)$ & $0.65(5)$ & 10.9 \\
\hline${ }^{42} \mathrm{Sc}$ & $3047.6(14)$ & $0.77(27)$ & $3069.2(85)$ & $0.72(6)$ & 3.1 \\
\hline${ }^{46} \mathrm{~V}$ & $3049.5(9)$ & $0.563(84)$ & $3075.1(32)$ & $0.71(6)$ & 1.3 \\
\hline${ }^{50} \mathrm{Mn}$ & $3048.4(12)$ & $0.476(71)$ & $3076.5(32)$ & $0.67(7)$ & 2.4 \\
\hline${ }^{54} \mathrm{Co}$ & $3050.8\left({ }_{-15}^{+11}\right)$ & $0.586(88)$ & $3075.6(36)$ & $0.75(8)$ & 1.3 \\
\hline${ }^{62} \mathrm{Ga}$ & $3074.1(15)$ & $0.78(12)$ & 3093.1(48) & $1.51(9)$ & 43.2 \\
\hline \multirow[t]{2}{*}{${ }^{74} \mathrm{Rb}$} & $3085(8)$ & $1.63(24)$ & $3078(12)$ & $1.86(27)$ & 0.3 \\
\hline & & \multicolumn{2}{|c|}{$\begin{aligned} \overline{\mathcal{F} t} & =3073.7(11) \\
\left|V_{\text {ud }}\right| & =0.97396(25) \\
& 0.99937(65)\end{aligned}$} & \multicolumn{2}{|c|}{$\begin{array}{c}\chi^{2}=69.5 \\
\chi_{d}^{2}=6.3\end{array}$} \\
\hline
\end{tabular}

Except for transitions ${ }^{14} \mathrm{O} \rightarrow{ }^{14} \mathrm{~N}$ and ${ }^{42} \mathrm{Sc} \rightarrow{ }^{42} \mathrm{Ca}$, all ISB corrections were calculated using the prescription sketched above. For the decay of a spherical nucleus ${ }^{14} \mathrm{O}$, the reference state is uniquely defined and thus the mixing of orientations was not necessary, whereas for that of ${ }^{42} \mathrm{Sc}$, an ambiguity of choosing its reference state is not related to the shape-current orientation. For both cases, the values and errors of $\delta_{\mathrm{C}}$ were taken from Ref. [15]. For the remaining cases, to account for uncertainties related to the basis size and collective-space cutoff, we assumed an error of $15 \%$. This is larger than the $10 \%$ uncertainties related to the basis size only, which were assumed in Ref. [15].

Systematic errors related to the form and parametrization of the functional itself were not included in the error budget. Moreover, similarly to our previous works [14,15], transition ${ }^{38} \mathrm{~K} \rightarrow{ }^{38} \mathrm{Ar}$ was disregarded. We recall that for this transition,

TABLE II. Similar as in Table I but for the transitions that are either unmeasured or measured with insufficient accuracy to be used for the SM tests.

\begin{tabular}{llcl}
\hline \hline $\begin{array}{l}\text { Parent } \\
\text { nucleus }\end{array}$ & $\delta_{\mathrm{C}}^{(\mathrm{SV})}$ & $\begin{array}{c}\text { Parent } \\
\text { nucleus }\end{array}$ & $\delta_{\mathrm{C}}^{(\mathrm{SV})}$ \\
\hline$T_{z}=-1:$ & $(\%)$ & $T_{z}=0:$ & \\
${ }^{18} \mathrm{Ne}$ & $1.37(21)$ & ${ }^{18} \mathrm{~F}$ & $1.22(18)$ \\
${ }^{26} \mathrm{Si}$ & $0.427(64)$ & ${ }^{22} \mathrm{Na}$ & $0.335(50)$ \\
${ }^{30} \mathrm{~S}$ & $1.24(19)$ & ${ }^{30} \mathrm{P}$ & $0.98(15)$ \\
\hline \hline
\end{tabular}


the calculated value of the ISB correction is unacceptably large because of a strong mixing of Nilsson levels originating from the $d_{3 / 2}$ and $s_{1 / 2}$ subshells. The problem can be partially cured by performing configuration-interaction calculations; see Ref. [18] and discussion in Sec. IV B.

To conform with the analyses of Hardy and Towner (HT) and Particle Data Group, the average value $\overline{\mathcal{F} t}=3073.7(11) \mathrm{s}$ was calculated using the Gaussian-distribution-weighted formula. This leads to the value of $\left|V_{\mathrm{ud}}\right|=0.97396(25)$, which is in very good agreement both with the Hardy and Towner result [30], $\left|V_{\mathrm{ud}}^{(\mathrm{HT})}\right|=0.97425(22)$, and central value obtained from the neutron decay $\left|V_{\mathrm{ud}}^{(v)}\right|=0.9746(19)$ [31]. By combining the value of $\left|V_{\mathrm{ud}}\right|$ calculated here with those of $\left|V_{\mathrm{us}}\right|=$ $0.2253(8)$ and $\left|V_{\mathrm{ub}}\right|=0.00413(49)$ of the 2014 Particle Data Group [3], one obtains

$$
\left|V_{\mathrm{ud}}\right|^{2}+\left|V_{\mathrm{us}}\right|^{2}+\left|V_{\mathrm{ub}}\right|^{2}=0.99937(65),
$$

which implies that the unitarity of the first row of the CKM matrix is satisfied with a precision better than $0.1 \%$. Note that, in spite of differences between individual values of $\delta_{\mathrm{C}}$, the values of $\overline{\mathcal{F} t}$ and $\left|V_{\text {ud }}\right|$ obtained here are in excellent agreement with the results of our previous works $[14,15]$.

The last two columns of Table I show results of the confidence-level (CL) test, as proposed in Ref. [29]. The CL test is based on the assumption that the CVC hypothesis is valid up to at least $\pm 0.03 \%$, which implies that a set of structure-dependent corrections should produce statistically consistent set of $\mathcal{F} t$ values. Assuming the validity of the calculated corrections $\delta_{\mathrm{NS}}$ [32], the empirical ISB corrections can be defined as

$$
\delta_{\mathrm{C}}^{(\text {exp })}=1+\delta_{\mathrm{NS}}-\frac{\overline{\mathcal{F} t}}{f t\left(1+\delta_{\mathrm{R}}^{\prime}\right)} .
$$

By the least-square minimization of the appropriate $\chi^{2}$, and treating the value of $\overline{\mathcal{F} t}$ as a single adjustable parameter, one can attempt to bring the set of empirical values $\delta_{\mathrm{C}}^{(\mathrm{exp})}$ as close as possible to the set of $\delta_{\mathrm{C}}$.

The empirical ISB corrections deduced in this way are tabulated in Table I. The table also lists individual contributions to the $\chi^{2}$ budget, whereas the total $\chi^{2}$ per degree of freedom $\left(\chi_{d}^{2}=\chi^{2} / n_{d}\right.$ for $\left.n_{d}=11\right)$ is $\chi_{d}^{2}=6.3$. This number is considerably smaller than the number quoted in our previous work [15], but much bigger than those obtained within (i) perturbative model reported in Ref. [29] (1.5), (ii) shell model with the Woods-Saxon radial wave functions (0.4) [28], (iii) shell model with Hartree-Fock radial wave functions (2.0) [33,34], (iv) Skyrme-Hartree-Fock with RPA (2.1) [35], and relativistic Hartree-Fock plus RPA model (1.7) [36]. It is worth stressing that, as before, our value of $\chi^{2} / n_{d}$ is deteriorated by two transitions that strongly violate the CVC hypothesis, ${ }^{62} \mathrm{Ga} \rightarrow{ }^{62} \mathrm{As}$ and ${ }^{34} \mathrm{Cl} \rightarrow{ }^{34} \mathrm{~S}$. These transitions give the $62 \%$ and $15 \%$ contributions to the total error budget, respectively. Without them, we would have obtained $\chi_{d}^{2}=$ $\chi^{2} / 9=1.7$.

\section{LOW-ENERGY SPECTRA OF SELECTED NUCLEI}

In this section, we present a short overview of results obtained using the NCCI approach. Because the model is based on simultaneous isospin and angular-momentum projections, it is particularly well suited to study $N \approx Z$ nuclei. These nuclei are of paramount importance for stringent many-body tests of the weak sector of the Standard Model [30,37]. In addition, they show specific structural features, like the Wigner energy or Nolen-Schiffer anomaly, which are difficult to reproduce within state-of-the-art nuclear models, in particular those rooted in a standard DFT.

A major goal of this work is to pin down strong and weak points of the NCCI approach proposed here. Hence, instead of performing a detailed study of a single nucleus, with many configurations being mixed, we decided to use a modest number of configurations and apply the model to a somewhat broad set of nuclei, starting from very light systems like ${ }^{6,8} \mathrm{Li}$ up to ${ }^{62} \mathrm{Zn}$. By adding additional configurations, the present results can certainly be refined. We believe, however, that such refinements will not affect the physical conclusions drawn in this work.

To efficiently track the MF configurations and to improve convergence properties of self-consistent calculations, all reference states used in the NCCI calculations below were determined assuming the conservation of parity and signature symmetries. For the $A \leqslant 42(A=62)$ nuclei, we employed the s.p. basis consisting of $N=10(12)$ spherical harmonic oscillator shells, respectively.

\section{A. Lithium isotopes: ${ }^{6} \mathrm{Li}$ and ${ }^{8} \mathrm{Li}$}

The Slater determinants, which we selected for the NCCI calculations in these two very light nuclei, are listed in Table III. For the sake of simplicity, the states are labeled by spherical quantum numbers $p_{1 / 2}$ or $p_{3 / 2}$ that dominate in the s.p. wave functions of the odd-proton and odd-neutron states. It turns out that such a labeling constitutes an intuitive and

TABLE III. Properties of the reference Slater determinants in ${ }^{6} \mathrm{Li}$ and ${ }^{8} \mathrm{Li}$, numbered by index $i$ and labeled by spherical quantum numbers of particle states above ${ }^{4} \mathrm{He}$. Listed are the $\mathrm{HF}$ energies $E_{\mathrm{HF}}$ in $\mathrm{MeV}$, quadrupole deformations $\beta_{2}$, triaxiality parameters $\gamma$, and neutron and proton s.p. alignments $j_{v}$ and $j_{\pi}$, together with their orientations $k$ in the intrinsic frame.

\begin{tabular}{lccccccc}
\hline \hline$i$ & $\left.{ }^{6} \mathrm{Li} ; i\right\rangle$ & $E_{\mathrm{HF}}$ & $\beta_{2}$ & $\gamma$ & $j_{v}$ & $j_{\pi}$ & $k$ \\
\hline 1 & $v p_{3 / 2} \otimes \pi p_{3 / 2}$ & -25.972 & 0.008 & $0^{\circ}$ & -0.50 & 1.50 & $\mathrm{Z}$ \\
2 & $v p_{3 / 2} \otimes \pi p_{3 / 2}$ & -26.787 & 0.330 & $0^{\circ}$ & 0.50 & 0.50 & $\mathrm{Z}$ \\
3 & $v p_{3 / 2} \otimes \pi p_{3 / 2}$ & -26.510 & 0.216 & $60^{\circ}$ & -1.50 & 1.50 & $\mathrm{Y}$ \\
4 & $v p_{3 / 2} \otimes \pi p_{3 / 2}$ & -27.244 & 0.207 & $60^{\circ}$ & 1.50 & 1.50 & $\mathrm{Y}$ \\
5 & $v p_{3 / 2} \otimes \pi p_{3 / 2}$ & -26.846 & 0.090 & $60^{\circ}$ & 1.50 & 0.50 & $\mathrm{Y}$ \\
$i$ & $\left.\left.\right|^{8} \mathrm{Li} ; i\right\rangle$ & $E_{\mathrm{HF}}$ & $\beta_{2}$ & $\gamma$ & $j_{v}$ & $j_{\pi}$ & $k$ \\
\hline 1 & $v p_{3 / 2} \otimes \pi p_{3 / 2}$ & -39.081 & 0.381 & $0^{\circ}$ & -1.50 & 0.50 & $\mathrm{Z}$ \\
2 & $v p_{1 / 2} \otimes \pi p_{3 / 2}$ & -34.041 & 0.361 & $0^{\circ}$ & 0.50 & 0.50 & $\mathrm{Z}$ \\
3 & $v p_{3 / 2} \otimes \pi p_{3 / 2}$ & -39.025 & 0.356 & $0^{\circ}$ & 1.50 & 0.50 & $\mathrm{Z}$ \\
4 & $v p_{3 / 2} \otimes \pi p_{3 / 2}$ & -35.680 & 0.027 & $0^{\circ}$ & -1.50 & 1.50 & $\mathrm{Z}$ \\
5 & $v p_{1 / 2} \otimes \pi p_{3 / 2}$ & -33.443 & 0.352 & $0^{\circ}$ & -0.50 & 0.50 & $\mathrm{Z}$ \\
\hline \hline
\end{tabular}




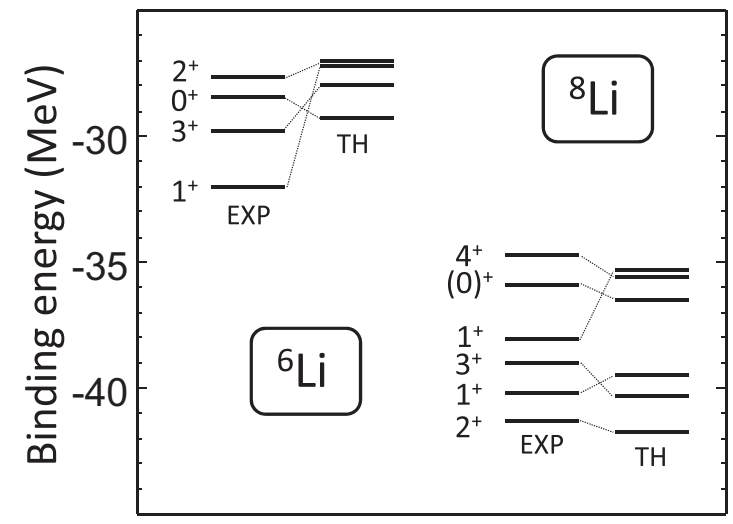

FIG. 3. Comparison between experimental and theoretical energy spectra of ${ }^{6} \mathrm{Li}$ and ${ }^{8} \mathrm{Li}$.

relatively unambiguous way to describe the configurations, even in cases of large deformations where the Nilsson picture formally prevails. The strategy behind selecting the reference configurations is to cover basic combinations of neutron or proton particle-hole $(\mathrm{p}-\mathrm{h})$ excitations having all possible alignments predicted by a simple $K$ scheme.

Results of our calculations are shown in Fig. 3. In the case of ${ }^{6} \mathrm{Li}$, theory clearly disagrees with data, with respect to both the ordering and values of energies. Let us first discuss the $T=0$ multiplet, composed of the $1^{+}$and $3^{+}$states. The ground state of ${ }^{6} \mathrm{Li}$ has quantum numbers $I=1^{+}, T=0$ and the experimental total energy of this state is $-31.995 \mathrm{MeV}$. In calculations, the lowest $I=1^{+}$state is placed above the lowest $I=0^{+}, T=1$ and $I=3^{+}, T=0$ solutions, and its energy of $-27.037 \mathrm{MeV}$ is almost $5 \mathrm{MeV}$ higher than in experiment. For comparison, the calculated energy of the $I=3^{+}, T=0$ member of the isoscalar multiplet is only $1.8 \mathrm{MeV}$ higher than in experiment. Hence, it is quite evident that the model lacks the isoscalar pairing $I=1, T=0$ correlations, cf. Ref. [38]. In the $N=Z$ nuclei, the model, or the underlying mean field, seems to favor the maximally aligned $T=0$ configurations. In Sec. IV C we demonstrate that the results obtained for ${ }^{42} \mathrm{Sc}$ corroborate these conclusions.

It is worth recalling here that in the context of searching for possible fingerprints of collective isoscalar $p n$-pairing phase in $N \approx Z$ nuclei, the isoscalar pairing, or deuteronlike correlations, were intensely discussed in the literature; see Refs. [39-43] and references cited therein. In particular, the isoscalar $p n$ pairing was considered to be the source of an additional binding energy that could offer a microscopic explanation of the so-called Wigner energy [44] - an extra binding energy along the $N=Z$ line, which is absent in the self-consistent MF mass models. In spite of numerous recent works following these early developments attempting to explain the isoscalar $p n$-pairing correlations and the Wigner energy (see Refs. [45-50] and references cited therein), the problem still lacks a satisfactory solution.

There are at least two major reasons for that: (i) an incompleteness of the HFB (HF) approaches used so far, which consider the $p n$ mixing only in the particle-particle channel (see discussion in Ref. [22]), and (ii) difficulties in evaluating the role of beyond-mean-field correlations. Recently, within the RPA including $p n$ correlations, the latter problem was addressed in Ref. [47]. Their systematic study of the isoscalar and isovector multiplets in magic and semimagic nuclei somewhat clearly indicated a missing relatively strong $T=0$ pairing. This seems to be in line with our NCCI model findings concerning description of $T=0, I=1$ states, but seems to contradict the conclusions of Refs. [46,48].

Concerning the $T=1$ multiplet consisting of the $0^{+}$and $2^{+}$ states, the theory tends to overbind the $0^{+}$state by $0.8 \mathrm{MeV}$ and underbind the $2^{+}$state by $0.4 \mathrm{MeV}$. This level of agreement is much better than the one obtained for the isoscalar multiplet. It should be rated as fair, but not fully satisfactory. It is, therefore, interesting and quite surprising to see that the addition of two neutrons in ${ }^{8} \mathrm{Li}$ seems to change the situation quite radically. Indeed, in this nucleus, for both the binding energies and distribution of levels below $5 \mathrm{MeV}$, the overall agreement between theory and experiment is very satisfactory, even if the calculated $1_{1}^{+}$and $3_{1}^{+}$states are interchanged; see Fig. 3 . The largest disagreement is obtained for the $1_{2}^{+}$state, where the theory underbinds experiment by almost $3 \mathrm{MeV}$. The states $0_{1}^{+}, 2_{2}^{+}$, and $4_{1}^{+}$are predicted at the excitation energies of 5.3, 4.7, and $6.2 \mathrm{MeV}$, respectively, in fair agreement with the data.

\section{B. $0^{+}$states in ${ }^{38} \mathrm{Ca},{ }^{38} \mathrm{~K}$, and ${ }^{38} \mathrm{Ar}$}

Recently, Park et al. [51] performed high-precision measurement of the superallowed $0^{+} \longrightarrow 0^{+}$Fermi decay of ${ }^{38} \mathrm{Ca} \rightarrow{ }^{38} \mathrm{~K}$; see also [52]. The reported $f t$ value of $3062.3(68) \mathrm{s}$ was measured with a relative precision of $\pm 0.2 \%$, which is sufficient for testing and determining the parameters of the electroweak sector of the Standard Model. This piece of data is the first, after almost a decade, addition to a set of canonical $0^{+} \longrightarrow 0^{+}$Fermi transitions, which are used to determine $\left|V_{u d}\right|$. Moreover, being a mirror partner to the superallowed $0^{+} \longrightarrow 0^{+}$Fermi transition ${ }^{38} \mathrm{~K} \rightarrow{ }^{38} \mathrm{Ar}$, it allows for sensitive tests of the ISB corrections and, in turn, for assessing the quality of nuclear models used to compute the ISBs [51].

Unfortunately, using the DFT with the SV Skyrme functional, which gives a strong mixing between the $2 s_{1 / 2}$ and $1 d_{3 / 2}$ orbits, it is difficult to determine the ISB corrections to the ${ }^{38} \mathrm{~K} \rightarrow{ }^{38} \mathrm{Ar}$ and ${ }^{38} \mathrm{Ca} \rightarrow{ }^{38} \mathrm{~K}$ superallowed transitions. In particular, in our previous static DFT calculations, the ISB corrections turned out to be of the order of $9 \%$, and thus were disregarded $[14,15]$.

In Ref. [18], we presented preliminary results of the NCCI study of ${ }^{38} \mathrm{Ca}$ and ${ }^{38} \mathrm{~K}$. Here we extend them to calculations that include three low-lying antialigned reference configurations in ${ }^{38} \mathrm{~K}$ and four configurations in both ${ }^{38} \mathrm{Ca}$ and ${ }^{38} \mathrm{Ar}$. Basic properties of these reference states are listed in Table IV.

Results of our NCCI calculations, including the binding energies of the lowest $0_{1}^{+}$states, excitation energies of the first excited $0_{2}^{+}$states, and the ISB corrections to superallowed $\beta$ decays, are visualized in Fig. 4. The total binding energies of the $0_{1}^{+}$states in these three nuclei are underestimated by circa $1 \%$. Concerning the first excited $\mathrm{O}_{2}^{+}$states, our model works very well in ${ }^{38} \mathrm{Ca}$. In this nucleus, the measured excitation energy, $\Delta E_{\mathrm{EXP}}=3057(18) \mathrm{keV}$, is only $186 \mathrm{keV}$ larger than 
TABLE IV. Similar as in Table III, but for ${ }^{38} \mathrm{~K},{ }^{38} \mathrm{Ca}$, and ${ }^{38} \mathrm{Ar}$. Here, the reference Slater determinants are labeled by the Nilsson quantum numbers pertaining to dominant components of the hole states below ${ }^{40} \mathrm{Ca}$. The first excited state in ${ }^{38} \mathrm{Ar}$, marked by asterisk, was converged with a weak quadrupole constraint.

\begin{tabular}{lccccccc}
\hline \hline$i$ & $|38 \mathrm{~K} ; i\rangle$ & $\Delta E_{\mathrm{HF}}$ & $\beta_{2}$ & $\gamma$ & $j_{\nu}$ & $j_{\pi}$ & $k$ \\
\hline 1 & $\left|202 \frac{3}{2}\right\rangle^{-2}$ & 0.000 & 0.083 & $60^{\circ}$ & -0.50 & 0.50 & $\mathrm{Y}$ \\
2 & $\left|220 \frac{1}{2}\right\rangle^{-2}$ & 1.380 & 0.035 & $0^{\circ}$ & 0.50 & -0.50 & $\mathrm{Z}$ \\
3 & $\left|211 \frac{1}{2}\right\rangle^{-2}$ & 1.559 & 0.042 & $0^{\circ}$ & -1.50 & 1.50 & $\mathrm{Z}$ \\
$i$ & $\left|{ }^{38} \mathrm{Ca} ; i\right\rangle$ & $\Delta E_{\mathrm{HF}}$ & $\beta_{2}$ & $\gamma$ & $j_{\nu}$ & $j_{\pi}$ & $k$ \\
\hline 1 & $\left|200 \frac{1}{2}\right\rangle^{-2}$ & 0.000 & 0.088 & $60^{\circ}$ & 0 & 0 & - \\
2 & $\left|200 \frac{1}{2}\right\rangle^{-2}$ & 0.762 & 0.006 & $0^{\circ}$ & 0 & 0 & - \\
3 & $\left|211 \frac{1}{2}\right\rangle^{-2}$ & 1.669 & 0.045 & $0^{\circ}$ & 0 & 0 & - \\
4 & $\left|220 \frac{1}{2}\right\rangle^{-1} \otimes\left|202 \frac{3}{2}\right\rangle^{-1}$ & 2.903 & 0.015 & $60^{\circ}$ & 0 & 0 & - \\
$i$ & $|38 \mathrm{Ar} ; i\rangle$ & $\Delta E_{\mathrm{HF}}$ & $\beta_{2}$ & $\gamma$ & $j_{\nu}$ & $j_{\pi}$ & $k$ \\
\hline 1 & $\left|200 \frac{1}{2}\right\rangle^{-2}$ & 0.000 & 0.088 & $60^{\circ}$ & 0 & 0 & - \\
2 & $\left|200 \frac{1}{2}\right\rangle^{-2}$ & $0.651^{(*)}$ & 0.002 & $46^{\circ}$ & 0 & 0 & - \\
3 & $\left|211 \frac{1}{2}\right\rangle^{-2}$ & 1.600 & 0.045 & $0^{\circ}$ & 0 & 0 & - \\
4 & $\left|220 \frac{1}{2}\right\rangle^{-1} \otimes\left|202 \frac{3}{2}\right\rangle^{-1}$ & 2.754 & 0.017 & $60^{\circ}$ & 0 & 0 & - \\
\hline \hline
\end{tabular}

the calculated one, $\Delta E_{\mathrm{TH}}=2871 \mathrm{keV}$. Note, however, that the calculated excitation energies of the $0_{2}^{+}$states are predicted to decrease with increasing $T_{z}$, at variance with the data. In turn, the difference between experimental and theoretical excitation energies of the $0_{2}^{+}$in ${ }^{38} \mathrm{Ar}$ grows to approximately $0.7 \mathrm{MeV}$.

The ISB corrections $\delta_{\mathrm{C}}$ to the ${ }^{38} \mathrm{Ca} \rightarrow{ }^{38} \mathrm{~K}$ transitions between the $0_{1}^{+} \rightarrow 0_{1}^{+}$and $0_{2}^{+} \rightarrow 0_{2}^{+}$states are equal to $1.7 \%$ and $1.5 \%$, respectively. As compared to our previous static model, which for the $0_{1}^{+}$states was giving an unacceptably large correction of $8.9 \%$, the NCCI result is strongly reduced. Nevertheless, it is still almost twice larger than that of Towner and Hardy [28], who quote the value of $0.77(7) \%$.

Similar results were obtained for the ${ }^{38} \mathrm{~K} \rightarrow{ }^{38} \mathrm{Ar}$ transitions, where the calculated corrections are $1.3 \%\left(0_{1}^{+} \rightarrow 0_{1}^{+}\right)$ and $1.4 \%\left(0_{2}^{+} \rightarrow 0_{2}^{+}\right)$. Again, as compared to the static variant of our model, the value for the $0_{1}^{+} \rightarrow 0_{1}^{+}$transition is strongly reduced, but it is considerably larger than the Towner and Hardy result of $0.66(6) \%$. Nevertheless, we see that the NCCI model removes, at least partially, pathologies encountered in the static variant.

\section{C. $\mathrm{A}=42$ nuclei: ${ }^{42} \mathrm{Sc}$ and ${ }^{42} \mathrm{Ca}$}

Within the conventional shell model, the ${ }^{42} \mathrm{Ca}$ and ${ }^{42} \mathrm{Sc}$ nuclei are treated as two-body systems above the core of ${ }^{40} \mathrm{Ca}$. Hence, they are often used by the shell-model community to adjust the isoscalar $T=0 ; I=1,3,5,7$, and isovector $T=1 ; I=0,2,4,6$ matrix elements within the $f_{7 / 2}$ shell. Here, we use these nuclei to test our NCCI model but, at least at this stage, without an intention of refitting the interaction. The aim of this exercise is to capture global trends and tendencies, which may allow us to identify systematic features of the NCCI model in describing these seemingly simple nuclei. From the

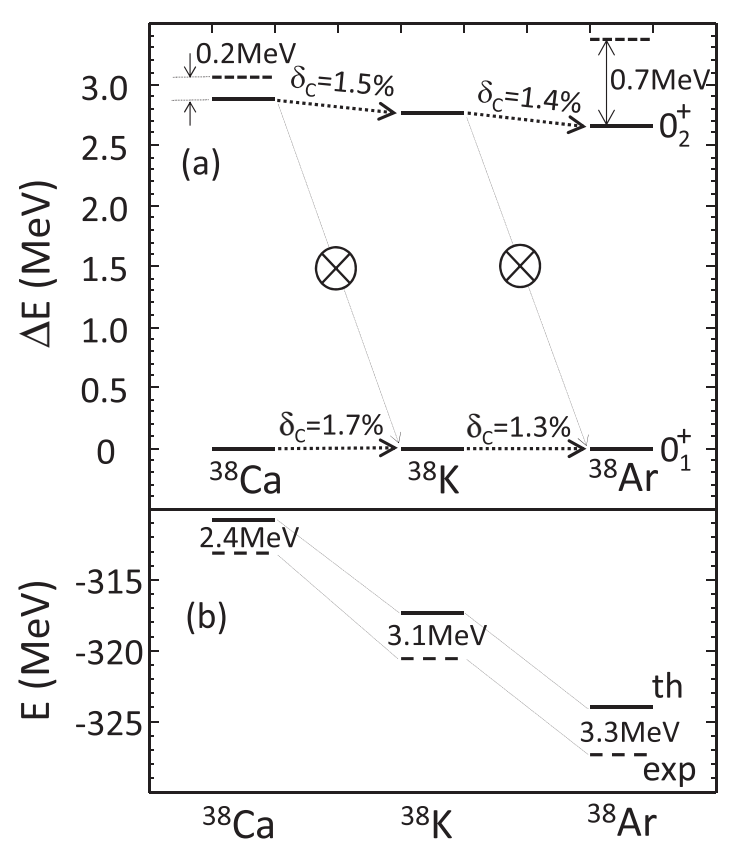

FIG. 4. (a) Excitation energies of the $0_{2}^{+}$states with respect to the $0_{1}^{+}$states in the $A=38$ isobaric triplet nuclei: $\mathrm{Ca}, \mathrm{K}$, and $\mathrm{Ar}$. Theoretical predictions and data [53] are shown with solid and dashed lines, respectively. Calculated values of $\delta_{\mathrm{C}}$ are also shown. Decays $0_{2}^{+} \rightarrow 0_{1}^{+}$indicated in the figure are predicted to be strongly hindered. (b) Comparison between the total binding energies of the $0_{1}^{+}$states ( $N=10$ harmonic oscillator shells were used).

perspective of our approach, such tests are by no means trivial, because these nuclei are here treated within the full corepolarization effects included; cf. discussion in Refs. [54,55].

The results of the NCCI calculations for the isovector and isoscalar multiplets in $A=42$ nuclei are depicted in Figs. 5 and 6 , and collected in Table $\mathrm{V}$. The reference states used in the calculation for ${ }^{42} \mathrm{Sc}$ are listed in Table VI. They cover all fully aligned $\left(K_{v}=K_{\pi}\right)$ states, which are almost purely

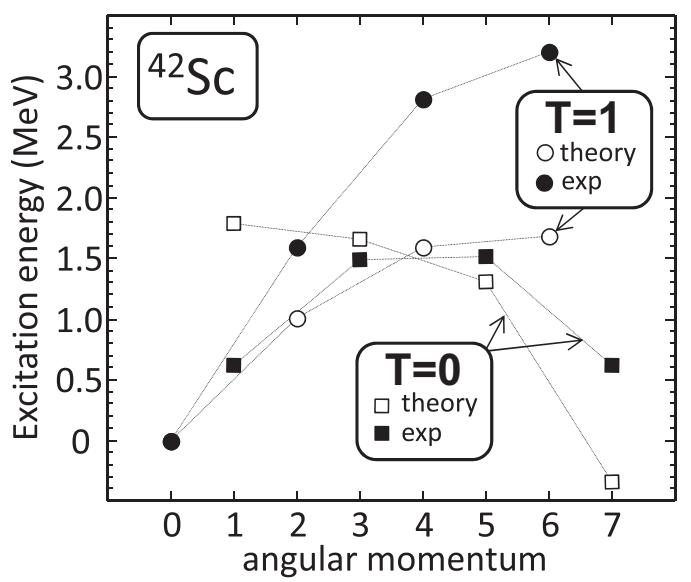

FIG. 5. Excitation energies of the isovector (circles) and isoscalar (squares) multiplets in ${ }^{42} \mathrm{Sc}$ with respect to the $0^{+}$state. Theoretical and experimental results are marked with open and solid symbols, respectively. 


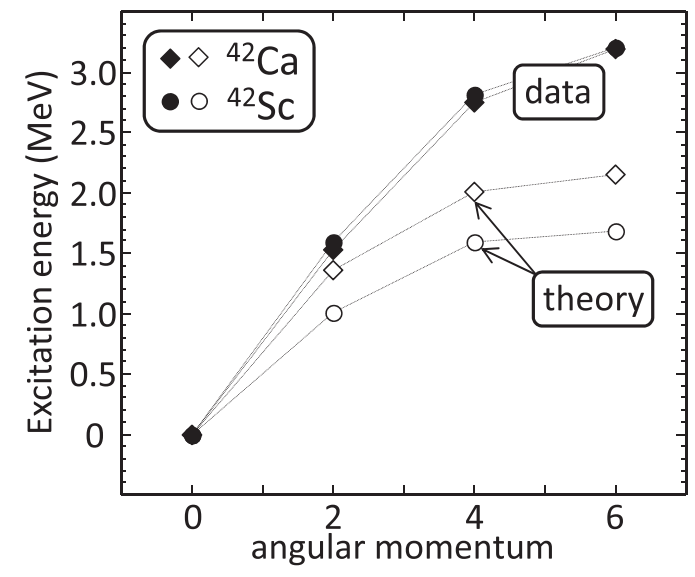

FIG. 6. Same as in Fig. 5, but for the isovector multiplets in ${ }^{42} \mathrm{Sc}$ (circles) and ${ }^{42} \mathrm{Ca}$ (diamonds).

isoscalar, all possible antialigned states $\left(K_{v}=-K_{\pi}\right)$, and two $K=1$ aligned states. The antialigned states manifestly violate the isospin symmetry and, as discussed in Ref. [56], are approximately 50-50 mixtures of the isoscalar and isovector components. The $K=1$ aligned states also violate the isospin symmetry.

The following three general conclusions can be drawn from the results presented in Fig. 5:

(i) The model lacks isoscalar pairing $T=0, I=1$ correlations. A similar deficiency was already observed in ${ }^{6} \mathrm{Li}, \mathrm{Sec}$. IV A.

(ii) The model strongly prefers fully aligned isoscalar $T=$ $0, I_{\max }=7$ states. Again, the conclusion is consistent with the one drawn from the calculated spectrum of ${ }^{6} \mathrm{Li}$.

(iii) The energy range spanned by the isovector states, $\Delta E_{T=1}=E_{T=1, I=6}-E_{T=1, I=0}$, is by a factor of two smaller in theory than in experiment. It is not obvious, however, whether this difference signalizes that the model underestimates the isovector pairing correla-

TABLE V. Excitation energies (total energies) of low-lying states (ground states) in ${ }^{42} \mathrm{Sc}$ and ${ }^{42} \mathrm{Ca}$. For ${ }^{42} \mathrm{Sc}$, we show calculated and experimental energies of the isovector $\left(I=0^{+}, 2^{+}, 4^{+}\right.$, and $\left.6^{+}\right)$and isoscalar $\left(I=1^{+}, 3^{+}, 5^{+}\right.$, and $\left.7^{+}\right)$multiplets. For ${ }^{42} \mathrm{Ca}$, we show the analogous energies of the isovector multiplet. All energies are in $\mathrm{MeV}$.

\begin{tabular}{lcccc}
\hline \hline & ${ }^{42} \mathrm{Sc}$ & ${ }^{42} \mathrm{Sc}$ & ${ }^{42} \mathrm{Ca}$ & ${ }^{42} \mathrm{Ca}$ \\
$I^{\pi}$ & $\Delta E_{\mathrm{I}}^{(\mathrm{th})}$ & $\Delta E_{\mathrm{I}}^{(\text {exp })}$ & $\Delta E_{\mathrm{I}}^{(\mathrm{th})}$ & $\Delta E_{\mathrm{I}}^{(\text {exp })}$ \\
\hline $0^{+}$ & -352.961 & -354.687 & -360.200 & -361.895 \\
$2^{+}$ & 1.012 & 1.586 & 1.357 & 1.525 \\
$4^{+}$ & 1.590 & 2.815 & 2.005 & 2.752 \\
$6^{+}$ & 1.696 & $(3.200)$ & 2.154 & 3.189 \\
$1^{+}$ & 1.785 & 0.611 & & \\
$3^{+}$ & 1.656 & 1.490 & & \\
$5^{+}$ & 1.336 & 1.510 & & \\
$7^{+}$ & -0.347 & 0.617 & & \\
\hline \hline
\end{tabular}

TABLE VI. Similar as in Table III, but for ${ }^{42} \mathrm{Sc}$. Here, the reference Slater determinants correspond to configurations $v f_{7 / 2} \otimes$ $\pi f_{7 / 2}$, and are labeled by intrinsic $K$ quantum numbers of valence neutrons and protons as $\left|\nu ; K_{\nu}\right\rangle \otimes\left|\pi ; K_{\pi}\right\rangle$. Reference states $i=1-4$ correspond to antialigned configurations, $K_{v}=-K_{\pi}$, thus carrying no net intrinsic alignment. Reference states $i=5-8$ represent aligned configurations, $K_{v}=K_{\pi}$, thus having the total alignments of 7, 5, 3 , and 1 , respectively. The remaining two configurations $i=9-10$ carry net alignments of 1 . The table also lists the HF energies $\Delta E_{\mathrm{I}=|\mathrm{K}|}$ relative to the $\left|v ; \frac{1}{2}\right\rangle \otimes\left|\overline{\pi ; \frac{1}{2}}\right\rangle$ solution. The last column shows excitation energy of the lowest $I=|K|$ state projected from a given Slater determinant.

\begin{tabular}{lccccr}
\hline \hline$i$ & $\left|{ }^{42} \mathrm{Sc} ; i\right\rangle$ & $\Delta E_{\mathrm{HF}}$ & $\beta_{2}$ & $\gamma$ & $\Delta E_{\mathrm{I}=|\mathrm{K}|}$ \\
\hline 1 & $\left|v ; \frac{1}{2}\right\rangle \otimes\left|\overline{\pi ; \frac{1}{2}}\right\rangle$ & 0.000 & 0.063 & 0 & 0.000 \\
2 & $\left|v ; \frac{3}{2}\right\rangle \otimes\left|\overline{\pi ; \frac{3}{2}}\right\rangle$ & 0.802 & 0.031 & 0 & 0.561 \\
3 & $\left|v ; \frac{5}{2}\right\rangle \otimes\left|\pi ; \frac{5}{2}\right\rangle$ & 0.986 & 0.008 & 60 & 0.551 \\
4 & $\left|v ; \frac{7}{2}\right\rangle \otimes\left|\pi ; \frac{7}{2}\right\rangle$ & 0.759 & 0.062 & 60 & 0.085 \\
5 & $\left|v ; \frac{7}{2}\right\rangle \otimes\left|\pi ; \frac{7}{2}\right\rangle$ & -0.929 & 0.061 & 60 & -0.647 \\
6 & $\left|v ; \frac{5}{2}\right\rangle \otimes\left|\pi ; \frac{5}{2}\right\rangle$ & 0.082 & 0.007 & 60 & 1.160 \\
7 & $\left|v ; \frac{3}{2}\right\rangle \otimes\left|\pi ; \frac{3}{2}\right\rangle$ & 0.345 & 0.032 & 0 & 1.594 \\
8 & $\left|v ; \frac{1}{2}\right\rangle \otimes\left|\pi ; \frac{1}{2}\right\rangle$ & 0.340 & 0.060 & 0 & 1.719 \\
9 & $\left|v ; \frac{3}{2}\right\rangle \otimes\left|\pi ;-\frac{1}{2}\right\rangle$ & 0.716 & 0.043 & 0 & 2.164 \\
10 & $\left|v ; \frac{5}{2}\right\rangle \otimes\left|\pi ;-\frac{3}{2}\right\rangle$ & 0.986 & 0.011 & 0 & 2.338 \\
\hline \hline
\end{tabular}

tions, overbinds the stretched (aligned) configurations, or both.

In the case of ${ }^{42} \mathrm{Ca}$, we focused on calculating the excitation energies of the $0^{+}$states, addressing, in particular, the question of structure and excitation energy of the intruder configuration. Experimentally, the intruder configuration is observed at very low excitation energy of $1.843 \mathrm{MeV}$; see Ref. [57] and references cited therein. In the calculations presented below we assumed that the structure of intruder state is associated with (multi)particle-(multi)hole excitations across the $N=Z=20$ magic gap, which in ${ }^{40} \mathrm{Ca}$ is of the order of $7.0 \mathrm{MeV}$; see Ref. [54] and references cited therein. The mechanism bringing the intruder configuration down in energy is sketched in Fig. 7.

The energy needed to elevate particles from the $d_{3 / 2}$ subshell to $f_{7 / 2}$ is at (near)spherical shape reduced by the energy associated with the spontaneous breaking of spherical symmetry in the intruder configuration, and further, by a rotational correction energy associated with the symmetry restoration. Owing to the configuration interaction, an additional gain in energy is expected too. The rotational correction and configuration interaction are also expected to lower slightly the MF g.s. energy. As shown in Fig. 7, the final value of the intruder excitation energy is an effect of a somewhat delicate interplay of several factors. Therefore, it is not surprising that the intruder states pose a real challenge for both the state-of-the-art nuclear shell models and MF-rooted theories.

In the NCCI calculations presented below, we mix states projected from the antialigned configurations that are listed in Table VII. The reference states can be divided into two 


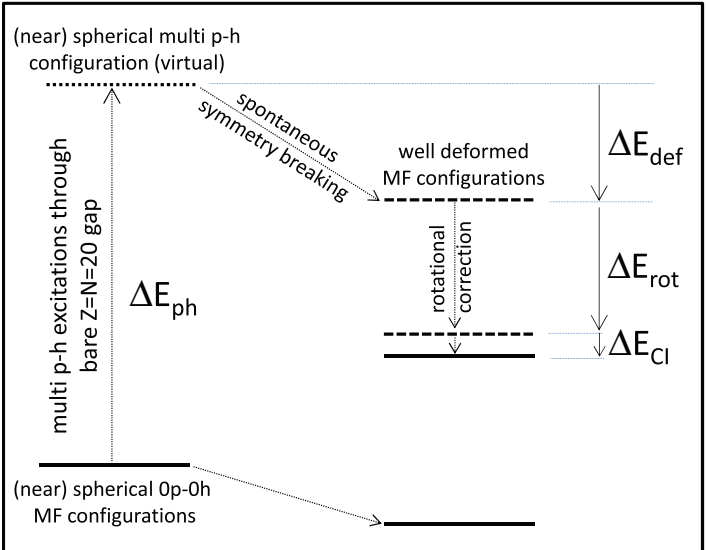

FIG. 7. Schematic illustration of the interplay between the primary physical ingredients contributing to the excitation energy of the intruder state within our model. See text for details.

classes. The first four configurations do not involve any cross-shell excitations. They correspond to the $|K\rangle \otimes|\bar{K}\rangle$ $0 \mathrm{p}-0 \mathrm{~h}\left(v f_{7 / 2}\right)^{2}$ configurations with magnetic quantum number of $K=1 / 2,3 / 2,5 / 2$, and $7 / 2$, respectively. The three remaining configurations are the lowest MF configurations involving two $f_{7 / 2}^{4} d_{3 / 2}^{-2}:\left(v f_{7 / 2}\right)^{2} \otimes\left(\pi f_{7 / 2}\right)^{2} \otimes\left(\pi d_{3 / 2}\right)^{-2}$, four $f_{7 / 2}^{6} d_{3 / 2}^{-4}:\left(v f_{7 / 2}\right)^{4} \otimes\left(v d_{3 / 2}\right)^{-2} \otimes\left(\pi f_{7 / 2}\right)^{2} \otimes\left(\pi d_{3 / 2}\right)^{-2}, \quad$ and six $\quad f_{7 / 2}^{8} d_{3 / 2}^{-6}:\left(v f_{7 / 2}\right)^{4} \otimes\left(v d_{3 / 2}\right)^{-2} \otimes\left(\pi f_{7 / 2}\right)^{4} \otimes\left(\pi d_{3 / 2}\right)^{-4}$ holes in $d_{3 / 2}$ shell, respectively.

The results of the NCCI calculations in ${ }^{42} \mathrm{Ca}$ are depicted in Figs. 6 and 8 and collected in Tables V and VII. Figure 6 shows the $I=0^{+}, 2^{+}, 4^{+}$, and $6^{+}$states-the isovector $T=1$ multiplet-obtained within the NCCI calculations involving only $\left(v f_{7 / 2}\right)^{2}$ reference states. The results are qualitatively similar to those in ${ }^{42} \mathrm{Sc}$. In both cases, theoretical spectra are compressed as compared to data. Detailed quantitative comparison reveals, however, surprisingly large differences between the theoretical and experimental spectra.

First, the energy differences $\delta E_{\mathrm{I}}=\Delta E_{I}\left({ }^{42} \mathrm{Ca}\right)-$ $\Delta E_{I}\left({ }^{42} \mathrm{Sc}\right)$ for $I=2^{+}, 4^{+}, 6^{+}$are positive (negative) in theory (experiment), respectively. Second, the absolute values of $\left|\delta E_{\mathrm{I}}\right|$ are a few times larger in theory as compared to the data.

TABLE VII. Similar as in Table III, but for ${ }^{42} \mathrm{Ca}$. Here, the Slater determinants are labeled by spherical quantum numbers pertaining to active neutron orbitals. The last column shows excitation energies of the lowest $0^{+}$states projected from a given Slater determinant.

\begin{tabular}{cccccc}
\hline \hline$i$ & $\left|{ }^{42} \mathrm{Ca} ; i\right\rangle$ & $\Delta E_{\mathrm{HF}}$ & $\beta_{2}$ & $\gamma$ & $\Delta E_{I=0}$ \\
\hline 1 & $\left|\frac{1}{2}\right\rangle \otimes\left|\frac{1}{2}\right\rangle$ & 0.000 & 0.069 & $0^{\circ}$ & 0.000 \\
2 & $\left|\frac{3}{2}\right\rangle \otimes\left|\frac{3}{2}\right\rangle$ & 0.516 & 0.033 & $0^{\circ}$ & 0.765 \\
3 & $\left|\frac{5}{2}\right\rangle \otimes\left|\frac{5}{2}\right\rangle$ & 0.544 & 0.007 & $60^{\circ}$ & 0.770 \\
4 & $\left|\frac{7}{2}\right\rangle \otimes\left|\frac{7}{2}\right\rangle$ & 0.084 & 0.061 & $60^{\circ}$ & 0.315 \\
5 & $f_{7 / 2}^{4} d_{3 / 2}^{-2}$ & 10.001 & 0.288 & $14^{\circ}$ & 6.860 \\
6 & $f_{7 / 2}^{6} d_{3 / 2}^{-4}$ & 10.986 & 0.414 & $22^{\circ}$ & 6.498 \\
7 & $f_{7 / 2}^{8} d_{3 / 2}^{-6}$ & 14.937 & 0.542 & $12^{\circ}$ & 9.619 \\
\hline \hline
\end{tabular}

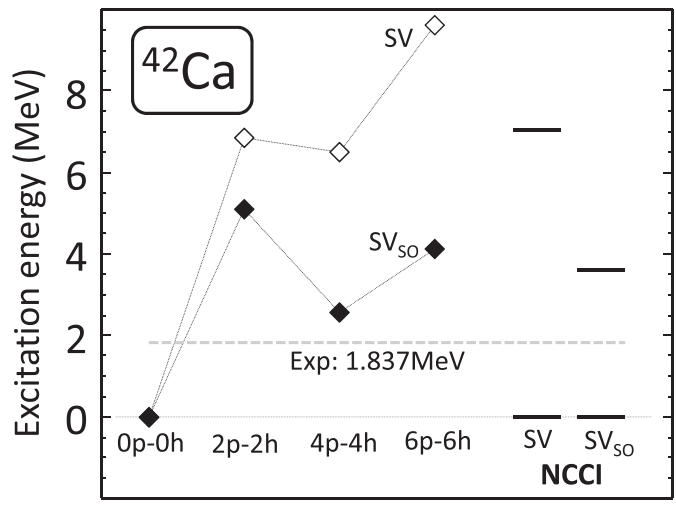

FIG. 8. The lowest $0^{+}$states projected from $\left(f_{7 / 2}\right)^{2}(0 \mathrm{p}-0 \mathrm{~h})$, $\left(f_{7 / 2}\right)^{4}\left(d_{3 / 2}\right)^{-2}(2 \mathrm{p}-2 \mathrm{~h}),\left(f_{7 / 2}\right)^{6}\left(d_{3 / 2}\right)^{-4}(4 \mathrm{p}-4 \mathrm{~h})$, and $\left(f_{7 / 2}\right)^{8}\left(d_{3 / 2}\right)^{-6}$ (6p-6h) reference states. Open (solid) diamonds refer to calculations performed using the $\mathrm{SV}$ and $\mathrm{SV}_{\text {So }}$ functionals, respectively. These results do not include configuration mixing. The right part shows excitation energies of the intruder state obtained within the NCCI theory with $\mathrm{SV}$ and $\mathrm{SV}_{\mathrm{SO}}$ interactions.

It means that the model tends to overestimate the ISB effects in clearly an unphysical manner. This influences the ISB correction to the $0^{+} \longrightarrow 0^{+}$Fermi $\beta$-decay matrix element, which in the present NCCI calculation rises to $\delta_{\mathrm{C}} \approx 2.2 \%$. Most likely, the unphysical component in the ISB effect is related to the time-odd polarizations and matrix elements originating from these fields, which are essentially absent in even-even systems. One should also remember that in the Skyrme functionals, including, of course, the SV force used here, the time-odd terms are very purely constrained.

Figure 8 shows the $0^{+}$states calculated using functionals $\mathrm{SV}$. The left part of the figure depicts the lowest $0^{+}$states projected from the reference states $\left(f_{7 / 2}\right)^{2}(0 \mathrm{p}-0 \mathrm{~h}),\left(f_{7 / 2}\right)^{4}\left(d_{3 / 2}\right)^{-2}$ (2p-2h), $\left(f_{7 / 2}\right)^{6}\left(d_{3 / 2}\right)^{-4}(4 \mathrm{p}-4 \mathrm{~h})$, and $\left(f_{7 / 2}\right)^{8}\left(d_{3 / 2}\right)^{-6}(6 \mathrm{p}-6 \mathrm{~h})$. These results do not include configuration mixing. Note, that symmetry restoration itself changes the optimal intruder configuration to $\left(v f_{7 / 2}\right)^{4} \otimes\left(\nu d_{3 / 2}\right)^{-2} \otimes\left(\pi f_{7 / 2}\right)^{2} \otimes\left(\pi d_{3 / 2}\right)^{-2}$ as compared to MF, which favors $\left(v f_{7 / 2}\right)^{2} \otimes\left(\pi f_{7 / 2}\right)^{2} \otimes$ $\left(\pi d_{3 / 2}\right)^{-2}$.

The right part of the figure shows excitation energies of the intruder states as obtained within the NCCI calculations. Here, all reference states listed in Table VII were included. For the SV force, the excitation energy of the lowest intruder configuration equals $7.5 \mathrm{MeV}$, and exceeds the data by 5.7 MeV. The main reason of the disagreement is related to an unphysically large $N=Z=20$ shell gap: The bare $N=20$ gap deduced directly from the s.p. HF levels in ${ }^{40} \mathrm{Ca}$ equals as much as $11.5 \mathrm{MeV}$. Its value exceeds the experimental gap by almost $4.5 \mathrm{MeV}$ (for an overview of experimental data, see Ref. [54] and references cited therein). It is therefore not surprising that the combined effects of deformation and rotational correction are unable to compensate for the large energy needed to lift the particles from the $d_{3 / 2}$ to $f_{7 / 2}$ shell (see Fig. 7).

To investigate interplay between the s.p. and collective effects, we repeated the NCCI calculations using the functional $\mathrm{SV}_{\mathrm{SO}}$, which differs from $\mathrm{SV}$ in a single aspect, namely, 
TABLE VIII. Same as in Table VII, but for the functional $\mathrm{SV}_{\text {so. }}$.

\begin{tabular}{cccccc}
\hline \hline$i$ & $\left|{ }^{42} \mathrm{Ca} ; i\right\rangle$ & $\Delta E_{\mathrm{HF}}$ & $\beta_{2}$ & $\gamma$ & $\Delta E_{I=0}$ \\
\hline 1 & $\left|\frac{1}{2}\right\rangle \otimes\left|\frac{1}{2}\right\rangle$ & 0.000 & 0.064 & $0^{\circ}$ & 0.000 \\
2 & $\left|\frac{1}{2}\right\rangle \otimes\left|\frac{1}{2}\right\rangle$ & 0.517 & 0.032 & $0^{\circ}$ & 0.679 \\
3 & $\left|\frac{1}{2}\right\rangle \otimes\left|\frac{1}{2}\right\rangle$ & 0.496 & 0.007 & $60^{\circ}$ & 0.676 \\
4 & $\left|\frac{1}{2}\right\rangle \otimes\left|\frac{1}{2}\right\rangle$ & 0.006 & 0.061 & $60^{\circ}$ & 0.200 \\
5 & $f_{7 / 2}^{4} d_{3 / 2}^{-2}$ & 8.399 & 0.276 & $15^{\circ}$ & 5.085 \\
6 & $f_{7 / 2}^{6} d_{3 / 2}^{-4}$ & 7.377 & 0.402 & $22^{\circ}$ & 2.548 \\
7 & $f_{7 / 2}^{8} d_{3 / 2}^{-6}$ & 9.955 & 0.532 & $15^{\circ}$ & 4.103 \\
\hline \hline
\end{tabular}

we increased its spin-orbit strength by a factor of 1.2. This readjustment allows one to reduce a disagreement between theoretical and experimental binding energies in $N \approx Z s d$ and lower- $p f$ shell nuclei to $\pm 1 \%$ level as shown in Ref. [58]. When applied to the heaviest $N=Z$ nucleus ${ }^{100} \mathrm{Sn}$ and its neighbor ${ }^{100} \mathrm{In}$ it gives $827.710 \mathrm{MeV}$ and $833.067 \mathrm{MeV}$ what is in an impressive agreement with the experimental binding energies equal $825.300 \mathrm{MeV}(833.110 \mathrm{MeV})$ in ${ }^{100} \mathrm{Sn}\left({ }^{100} \mathrm{In}\right)$, respectively. Ability to reproduce masses is among the most important indicators of a quality of DFT-based models. Such a readjustment of the SO strength is also the simplest and most efficient mechanism allowing us to reduce the magic $Z=N=20$ gap [59]. For the $\mathrm{SV}_{\mathrm{SO}}$ force, the bare gap equals $9.6 \mathrm{MeV}$, which is by almost $1.9 \mathrm{MeV}$ smaller than the original $\mathrm{SV}$ gap, but still it is much larger, by circa $2.6 \mathrm{MeV}$, than the experimental value. Results of the NCCI calculations obtained using functional $\mathrm{SV}_{\mathrm{SO}}$ are shown in Fig. 8. Now the projected and NCCI calculations both favor the configuration $\left(\nu f_{7 / 2}\right)^{4} \otimes$ $\left(\nu d_{3 / 2}\right)^{-2} \otimes\left(\pi f_{7 / 2}\right)^{2} \otimes\left(\pi d_{3 / 2}\right)^{-2}$. We also note that for both the $\mathrm{SV}$ and $\mathrm{SV}_{\mathrm{SO}}$ functionals, geometrical properties of the reference states (deformations) are very similar.

When discussing the influence of various effects on the final position of the intruder state, it is worth stressing the role of the symmetry restoration. The rotational correction lowers the intruder state by $4.9 \mathrm{MeV}$, bringing its excitation energy to $2.3 \mathrm{MeV}$, which is only $0.5 \mathrm{MeV}$ above the experiment. However, after the configuration mixing, the excitation energy of the intruder state increases to about 3.6 MeV, that is, it becomes again $1.7 \mathrm{MeV}$ higher as compared to data. This is because of the configuration mixing in the ground state, which lowers its energy by almost $1 \mathrm{MeV}$, whereas it leaves the position of the intruder state almost unaffected. The reason for that is the fact that the $\left(v f_{7 / 2}\right)^{2}$ antialigned reference states (states $1-4$ in Tables VII and VIII) are almost linearly dependent and thus mix relatively strongly. Conversely, at deformations corresponding to the intruder configuration $\left(v f_{7 / 2}\right)^{4} \otimes\left(\nu d_{3 / 2}\right)^{-2} \otimes\left(\pi f_{7 / 2}\right)^{2} \otimes$ $\left(\pi d_{3 / 2}\right)^{-2}$, the Nilsson scheme prevails. Therefore, the intruder configurations become almost linearly independent and appear to mix very weakly. The amount of the mixing was tested by performing additional calculations of matrix elements between the lowest $\left(v f_{7 / 2}\right)^{4} \otimes\left(\nu d_{3 / 2}\right)^{-2} \otimes\left(\pi f_{7 / 2}\right)^{2} \otimes\left(\pi d_{3 / 2}\right)^{-2}$ configuration and the excited configurations involving the same number of $\left(d_{3 / 2}\right)^{-4}$ holes. All these matrix elements turned out to be negligibly small.

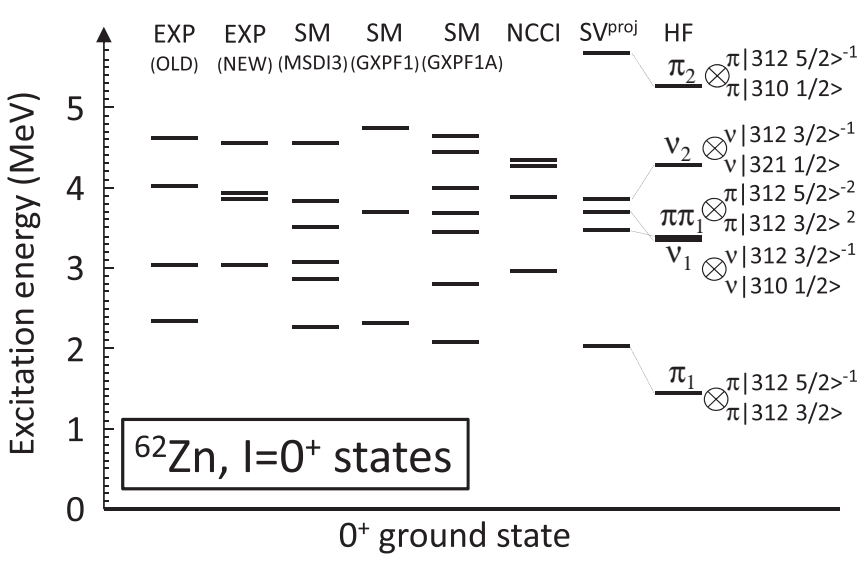

FIG. 9. The low-lying $0^{+}$states in ${ }^{62} \mathrm{Zn}$. The first two columns show old and new experimental data; see [60] for details. The next three columns collect the results of the shell-model calculations using interactions MSDI3 [61], GXPF1 [62], and GXPF1A [63], respectively. The last three columns show results obtained within the NCCI approach, angular-momentum projection, and pure HF method, respectively. From Ref. [18].

\section{D. $A=62$ nuclei: ${ }^{62} \mathrm{Zn}$ and ${ }^{62} \mathrm{Ga}$}

For ${ }^{62} \mathrm{Zn}$, the results of the NCCI calculations of the lowlying $0^{+}$states were communicated in Ref. [18]. Here, for the sake of completeness, we briefly summarize the results obtained therein. The calculated spectrum of the $0^{+}$states below the excitation energy of $5 \mathrm{MeV}$ is shown in Fig. 9. The NCCI calculations were based on six reference states that include: the ground state, the two lowest neutron $\mathrm{p}-\mathrm{h}$ excitations $v_{1}$ and $v_{2}$, the two lowest proton $\mathrm{p}$-h excitations $\pi_{1}$ and $\pi_{2}$, and the lowest proton $2 \mathrm{p}-2 \mathrm{~h}$ excitation $\pi \pi_{1}$. Their properties are listed in Table IX.

As discussed in Ref. [18], the calculated spectrum of $0^{+}$states is in a very good agreement with the recent data communicated by Leach et al. [60]. As shown in Fig. 10(a), the calculated total g.s. energy is stable with increasing the number of reference configurations. Its value of $-526.595 \mathrm{MeV}(N=$ 12 harmonic oscillator shells were used) underestimates the experiment by roughly $2 \%$.

In spite of the fact that the total binding energy is relatively stable, the calculated ISB corrections to the superallowed transition ${ }^{62} \mathrm{Ga} \rightarrow{ }^{62} \mathrm{Zn}$ strongly depend on the details of the

TABLE IX. Similar as in Table III, but for ${ }^{62} \mathrm{Zn}$. Here, the Slater determinants are labeled by neutron and proton configurations described in the text. The last column shows energies of the lowest $0^{+}$states projected from a given Slater determinant.

\begin{tabular}{lcrcccccr}
\hline \hline$i$ & $\left|{ }^{62} \mathrm{Zn} ; i\right\rangle$ & \multicolumn{1}{c}{$\Delta E_{\mathrm{HF}}$} & $\beta_{2}$ & $\gamma$ & $j_{v}$ & $j_{\pi}$ & \multicolumn{1}{c}{$k$} & \multicolumn{1}{c}{$\Delta E_{\mathrm{I}=0}$} \\
\hline 1 & g.s. & -521.549 & 0.270 & $31^{\circ}$ & 0.000 & 0.000 & -526.405 \\
2 & $\pi_{1}$ & 1.433 & 0.286 & $20^{\circ}$ & 0.005 & $0.152 \mathrm{Y}$ & 2.036 \\
3 & $v_{1}$ & 3.347 & 0.255 & $40^{\circ}$ & 0.689 & $0.318 \mathrm{X}$ & 3.703 \\
4 & $v_{2}$ & 4.287 & 0.240 & $25^{\circ}$ & -0.281 & $-0.325 \mathrm{Y}$ & 3.852 \\
5 & $\pi_{2}$ & 5.251 & 0.246 & $48^{\circ}$ & -0.103 & $-0.076 \mathrm{X}$ & 5.672 \\
6 & $\pi \pi_{1}$ & 3.381 & 0.251 & $38^{\circ}$ & 0.000 & 0.000 & 3.471 \\
\hline \hline
\end{tabular}




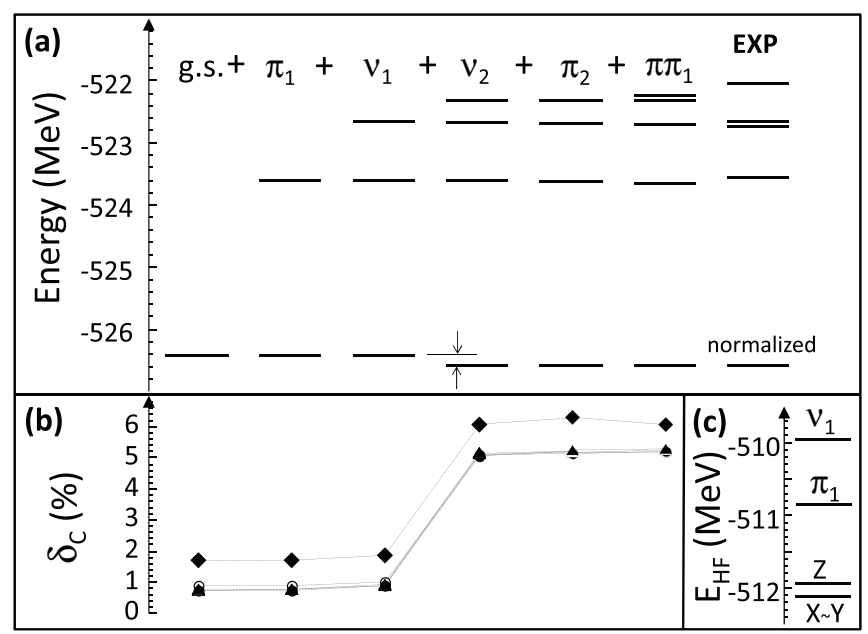

FIG. 10. (a) The low-lying $0^{+}$states in ${ }^{62} \mathrm{Zn}$ in the function of the number of configurations included in the NCCI calculations. (b) Calculated ISB corrections versus the number of configurations taken into account in the daughter nucleus. Different curves correspond to different sets of configurations taken to calculate the $0^{+}$state in ${ }^{62} \mathrm{Ga}$. (c) The HF energies of configurations included in the calculation of ${ }^{62} \mathrm{Ga}$; see Table X. Further details are given in the text.

calculation. This is illustrated in Fig. 10(b), which shows values of $\delta_{\mathrm{C}}$ in function of the number of configurations taken for the NCCI calculations in the daughter nucleus ${ }^{62} \mathrm{Zn}$. The four different curves correspond to different model spaces taken for the NCCI calculation in the parent nucleus ${ }^{62} \mathrm{Ga}$; see Table $\mathrm{X}$ and Fig. 10(c). In terms of Nilsson numbers, counted relatively to the ${ }^{64} \mathrm{Zn}_{32}$ even-even core, the configurations $\mathrm{X}, \mathrm{Y}, \mathrm{Z}$ correspond to differently aligned $\nu|3123 / 2\rangle^{-1} \otimes \pi|3123 / 2\rangle^{-1}$ two-hole states, $\pi_{1}$ denotes $v|3123 / 2\rangle^{-1} \otimes \pi|3125 / 2\rangle^{-1}$, two-hole state while $v_{1}$ is $v|3211 / 2\rangle^{-1} \otimes \pi|3123 / 2\rangle^{-1}$. The three curves labeled with open dots, and open and solid triangles correspond to states $0^{+}$projected from the $[\mathrm{X}, \mathrm{Y}]$, $[\mathrm{X}, \mathrm{Y}, \mathrm{Z}]$, and $\left[\mathrm{X}, \mathrm{Y}, \mathrm{Z}, \pi_{1}\right]$ configurations, respectively. These curves essentially overlap with each other, thus showing no influence of the configuration mixing (in this restricted model space) on the structure of the $0^{+}$state in the parent nucleus. Note, however, that an extension of the model space by adding the lowest neutron $\mathrm{p}-\mathrm{h}$ excitation, $\left[\mathrm{X}, \mathrm{Y}, \mathrm{Z}, \pi_{1}, \nu_{1}\right]$, leads to an increase in $\delta_{\mathrm{C}}$ of about $1 \%$. Note also, that all curves are particularly sensitive to an admixture of the $v_{2}$ configuration in the daughter nucleus. This admixture increases $\delta_{\mathrm{C}}$ by almost $4 \%$. The analysis clearly shows that, within the present

TABLE X. Same as in Table IX, but for ${ }^{62} \mathrm{Ga}$.

\begin{tabular}{ccrcccccr}
\hline \hline$i$ & $\left|{ }^{62} \mathrm{Ga} ; i\right\rangle$ & \multicolumn{1}{c}{$\Delta E_{\mathrm{HF}}$} & $\beta_{2}$ & $\gamma$ & $j_{v}$ & $j_{\pi}$ & $k$ & $\Delta E_{\mathrm{I}=0}$ \\
\hline 1 & $\mathrm{Y}$ & -512.122 & 0.268 & $30^{\circ}$ & -0.138 & $0.149 \mathrm{Y}$ & -516.930 \\
2 & $\mathrm{X}$ & 0.007 & 0.268 & $30^{\circ}$ & 0.180 & $-0.170 \mathrm{X}$ & -0.001 \\
3 & $\mathrm{Z}$ & 0.190 & 0.269 & $30^{\circ}$ & -0.299 & $0.264 \mathrm{Z}$ & 0.005 \\
4 & $\pi_{1}$ & 1.266 & 0.284 & $20^{\circ}$ & -0.012 & $-0.264 \mathrm{X}$ & 2.175 \\
5 & $v_{1}$ & 1.977 & 0.255 & $35^{\circ}$ & -0.440 & $-0.351 \mathrm{X}$ & 3.151 \\
\hline \hline
\end{tabular}

implementation of the model, it is essentially impossible to match the spaces of states used to calculate the parent and daughter nuclei. The reasons are manifold. The lack of representability of the $T=1, I=0$ states in the $N=Z$ nucleus within the conventional MF using products of neutron and proton wave functions and difficulties in constraining the time-odd part of the functional are two of them. Difficulty of matching the model spaces in the parent and daughter nuclei introduce here an artificial ISB effect. As a result, beyond a simple mixing of orientations used in the result given in Table I, the NCCI approach cannot be used for determining the ISB corrections to the transition ${ }^{62} \mathrm{Ga} \rightarrow{ }^{62} \mathrm{Zn}$.

\section{SUMMARY AND PERSPECTIVES}

In this work, we introduced the NCCI model involving the isospin and angular-momentum projections and subsequent mixing of states having good angular momentum and properly treated Coulomb isospin mixing. The model is capable of treating rigorously both the fundamental (spherical, particle number) as well as approximate (isospin) nuclear symmetries. Its potentially unrestricted range of applicability and a natural ability to treat the core-polarization effects resulting from a subtle interplay between the long-range Coulomb force and short-range hadronic nucleon-nucleon forces, which are treated on the same footing, makes it an interesting alternative to the nuclear shell model.

The NCCI model employs states projected from the lowlying (multi)particle-(multi)hole deformed Slater determinants (configurations) calculated self-consistently using the HartreeFock method. In the present realization, the same SV Skyrme functional was used both to compute the configurations and to mix the states. This restriction, however, can be easily relaxed opening a room for various generalizations of the model. In particular, one can attempt to correct an interaction used at the mixing stage to improve a description of $T=0, I=1^{+}$states in ${ }^{6} \mathrm{Li}$ and ${ }^{42} \mathrm{Sc} N=Z$ nuclei.

We demonstrated that our NCCI formalism is capable of capturing many features of the low-lying energy spectra in such diverse systems as ${ }^{8} \mathrm{Li}, A=38$ isospin triplet nuclei or ${ }^{62} \mathrm{Zn}$ and ${ }^{62} \mathrm{Ga}$ nuclei. A reasonable agreement with experiment was obtained when using a relatively small number of configurations, which supports our claims that the model can indeed be applicable to medium heavy nuclei with an affordable numerical cost. Our recent systematic study of Gamow-Teller matrix elements in $T_{z}=1 / 2 s d$ - and lower $p f$-shell mirror nuclei performed in Ref. [58] (see also [64]) confirms that the model can incorporate in a controlled way many important correlations into the nuclear wave function.

Finally, we also calculated the new set of the ISB corrections to superallowed $T=1, I=0^{+} \rightarrow T=1, I=0^{+}$beta transitions. The refined corrections are collected in Table I for a canonical set of precisely measured transitions and in Table II for transitions that were either unmeasured or measured with the accuracy insufficient for the Standard Model tests. These results are based on mixing the $I=0^{+}$states projected from the so-called $X, Y$, and $Z$ configurations corresponding to different shape-current orientations in odd-odd nuclei. Unfortunately, an attempt to perform more advanced calculation 
for the transition ${ }^{62} \mathrm{Ga} \rightarrow{ }^{62} \mathrm{Zn}$, which would take into account more configurations, failed because of difficulties in matching the model spaces in even-even and odd-odd nuclei.

\section{ACKNOWLEDGMENTS}

This work was supported in part by the Polish National Science Centre (NCN) under Contracts No.
2012/07/B/ST2/03907 and No. 2014/15/N/ST2/03454, by the THEXO JRA within the EU-FP7-IA project ENSAR (Grant No. 262010), by the ERANET-NuPNET grant SARFEN of the Polish National Centre for Research and Development (NCBiR), and by the Academy of Finland and University of Jyväskylä within the FIDIPRO programme. We acknowledge the CSC-IT Center for Science Ltd., Finland, for the allocation of computational resources.
[1] N. Cabibbo, Phys. Rev. Lett. 10, 531 (1963).

[2] M. Kobayashi and T. Maskawa, Prog. Theor. Phys. 49, 652 (1973).

[3] K. A. Olive and Particle Data Group, Chin. Phys. C 38, 090001 (2014).

[4] I. S. Towner and J. C. Hardy, Rep. Prog. Phys. 73, 046301 (2010).

[5] W. Satuła, J. Dobaczewski, W. Nazarewicz, and M. Rafalski, Phys. Rev. Lett. 103, 012502 (2009).

[6] P. Hohenberg and W. Kohn, Phys. Rev. 136, B864 (1964).

[7] W. Kohn and L. Sham, Phys. Rev. 140, A1133 (1965).

[8] J. Blaizot and G. Ripka, Quantum theory of Finite Systems (MIT Press, Cambridge, 1986).

[9] D. Lacroix, T. Duguet, and M. Bender, Phys. Rev. C 79, 044318 (2009).

[10] M. Bender, T. Duguet, and D. Lacroix, Phys. Rev. C 79, 044319 (2009).

[11] W. Satuła and J. Dobaczewski, Phys. Rev. C 90, 054303 (2014).

[12] J. Dobaczewski, J. Phys. G: Nucl. Part. Phys. 43, 04LT01 (2016).

[13] M. Beiner, H. Flocard, N. V. Giai, and P. Quentin, Nucl. Phys. A 238, 29 (1975).

[14] W. Satuła, J. Dobaczewski, W. Nazarewicz, and M. Rafalski, Phys. Rev. Lett. 106, 132502 (2011).

[15] W. Satuła, J. Dobaczewski, W. Nazarewicz, and T. R. Werner, Phys. Rev. C 86, 054316 (2012).

[16] W. Satuła, J. Dobaczewski, M. Konieczka, and W. Nazarewicz, Invited talk presented by WS at the EURISOL User Group Topical Meeting 2013, July 1-3, Krakow, Poland, arXiv:1307.1550.

[17] W. Satuła, J. Dobaczewski, M. Konieczka, and W. Nazarewicz, Acta Phys. Pol. B 45, 167 (2014).

[18] W. Satuła, J. Dobaczewski, and M. Konieczka, JPS Conf. Proc. 6, 020015 (2015).

[19] B. Bally, B. Avez, M. Bender, and P.-H. Heenen, Phys. Rev. Lett. 113, 162501 (2014).

[20] B. Barrett, P. Navrátil, and J. Vary, Prog. Part. Nucl. Phys. 69, 131 (2013).

[21] R. J. Bartlett, Mol. Phys. 108, 3299 (2010).

[22] K. Sato, J. Dobaczewski, T. Nakatsukasa, and W. Satuła, Phys. Rev. C 88, 061301(R) (2013).

[23] P. Ring and P. Schuck, The Nuclear Many-Body Problem (Springer-Verlag, Berlin, 1980).

[24] J. Dobaczewski, W. Satuła, B. Carlsson, J. Engel, P. Olbratowski, P. Powałowski, M. Sadziak, J. Sarich, N. Schunck, A. Staszczak, M. Stoitsov, M. Zalewski, and H. Zduńczuk, Comput. Phys. Commun. 180, 2361 (2009).

[25] W. Satuła et al. (unpublished).

[26] N. Schunck, J. Dobaczewski, J. McDonnell, W. Satuła, J. Sheikh, A. Staszczak, M. Stoitsov, and P. Toivanen, Comput. Phys. Commun. 183, 166 (2012).
[27] N. Schunck et al. (unpublished).

[28] I. S. Towner and J. C. Hardy, Phys. Rev. C 77, 025501 (2008).

[29] I. S. Towner and J. C. Hardy, Phys. Rev. C 82, 065501 (2010).

[30] J. C. Hardy and I. S. Towner, J. Phys. G: Nucl. Part. Phys. 41, 114004 (2014).

[31] K. Nakamura (Particle Data Group), J. Phys. G 37, 075021 (2010).

[32] I. S. Towner, Phys. Lett. B 333, 13 (1994).

[33] W. E. Ormand, Phys. Rev. C 53, 214 (1996).

[34] J. C. Hardy and I. S. Towner, Phys. Rev. C 79, 055502 (2009).

[35] H. Sagawa, N. Van Giai, and T. Suzuki, Phys. Rev. C 53, 2163 (1996).

[36] H. Liang, N. V. Giai, and J. Meng, Phys. Rev. C 79, 064316 (2009).

[37] O. Naviliat-Cuncic and N. Severijns, Phys. Rev. Lett. 102, 142302 (2009).

[38] L. Coraggio, A. Covello, A. Gargano, and N. Itaco, Phys. Rev. C 88, 041304 (2013).

[39] A. L. Goodman, Nucl. Phys. A 186, 475 (1972).

[40] J. Engel, K. Langanke, and P. Vogel, Phys. Lett. B 389, 211 (1996).

[41] W. Satuła and R. Wyss, Phys. Lett. B 393, 1 (1997).

[42] W. Satuła and R. Wyss, Phys. Rev. Lett. 86, 4488 (2001).

[43] W. Satuła and R. Wyss, Phys. Rev. Lett. 87, 052504 (2001).

[44] W. Satuła, D. Dean, J. Gary, S. Mizutori, and W. Nazarewicz, Phys. Lett. B 407, 103 (1997).

[45] N. Sandulescu, D. Negrea, and C. W. Johnson, Phys. Rev. C 86, 041302(R) (2012).

[46] I. Bentley and S. Frauendorf, Phys. Rev. C 88, 014322 (2013).

[47] B. G. Carlsson and J. Toivanen, Phys. Rev. C 89, 054324 (2014).

[48] I. Bentley, K. Neergard, and S. Frauendorf, Phys. Rev. C 89, 034302 (2014).

[49] M. Sambataro and N. Sandulescu, Phys. Rev. C 91, 064318 (2015).

[50] D. S. Delion and V. V. Baran, Phys. Rev. C 91, 024312 (2015).

[51] H. I. Park, J. C. Hardy, V. E. Iacob, M. Bencomo, L. Chen, V. Horvat, N. Nica, B. T. Roeder, E. Simmons, R. E. Tribble, and I. S. Towner, Phys. Rev. Lett. 112, 102502 (2014).

[52] B. Blank, J. Thomas, P. Ascher, L. Audirac, A. Bacquias, L. Cáceres, G. Canchel, L. Daudin, F. de Oliveira Santos, F. Didierjean, M. Gerbaux, J. Giovinazzo, S. Grévy, T. K. Nieto, I. Matea, F. Munoz, M. Roche, L. Serani, N. Smirnova, and J. Souin, Eur. Phys. J. A 51, 8 (2015).

[53] Evaluated Nuclear Structure Data File.

[54] M. Zalewski, J. Dobaczewski, W. Satuła, and T. R. Werner, Phys. Rev. C 77, 024316 (2008).

[55] D. Tarpanov, J. Toivanen, J. Dobaczewski, and B. G. Carlsson, Phys. Rev. C 89, 014307 (2014).

[56] W. Satuła, J. Dobaczewski, W. Nazarewicz, and M. Rafalski, Phys. Rev. C 81, 054310 (2010). 
[57] K. Hadyńska-Klęk et al., Acta Phys. Pol. B 44, 617 (2013).

[58] M. Konieczka, P. Bączyk, and W. Satuła, Phys. Rev. C 93, 042501 (2016).

[59] H. Zduńczuk, W. Satuła, and R. A. Wyss, Phys. Rev. C 71, 024305 (2005).

[60] K. Leach et al., Phys. Rev. C 88, 031306(R) (2013).
[61] J. Koops and W. Glaudemans, Z. Phys. A 280, 181 (1977).

[62] M. Honma, T. Otsuka, B. A. Brown, and T. Mizusaki, Phys. Rev. C 65, 061301(R) (2002).

[63] M. Honma, T. Otsuka, B. A. Brown, and T. Mizusaki, Phys. Rev. C 69, 034335 (2004).

[64] W. Satuła and W. Nazarewicz, Phys. Scr. 91, 023013 (2016). 